CUBO A Mathematical Journal

Vol.19, No 01, (89-110). March 2017

\title{
Weighted pseudo Almost periodic solutions for fractional order stochastic impulsive differential equations
}

\author{
Vikram Singh and DwiJendra N Pandey \\ Department of Mathematics, \\ Indian Institute of Technology Roorkee \\ Roorkee-247667, India. \\ vikramiitr1@gmail.com, dwij.iitk@gmail.com
}

\begin{abstract}
In this paper, we deal with the existence and uniqueness of piecewise square mean weighted pseudo almost periodic solutions for a class of fractional order stochastic impulsive differential equations. The working tools are based on fixed point technique, fractional power operators and stochastic analysis; methods and theory are adopted from deterministic fractional systems. In addition, an example is given to illustrate the theory.
\end{abstract}

\section{RESUMEN}

En este artículo estudiamos la existencia y unicidad de soluciones pseudo casi periódicas con pesos promedio cuadrado a trozos para una clase de ecuaciones diferenciales estocásticas impulsivas de orden fraccional. Las herramientas de trabajo están basadas en la técnica de punto fijo, operadores de potencia fraccional y análisis estocástico; los métodos y teoría están adaptados a partir de sistemas fraccionales deterministas. Adicionalmente, damos un ejemplo para ilustrar la teoría.

Keywords and Phrases: Fractional stochastic impulsive differential equation, Square-mean piecewise weighted pseudo almost periodicity, Analytic semigroup, Fractional power operator.

2010 AMS Mathematics Subject Classification: 26A33, 34A37, 34C27, 34G20, 35R12, 35R60, 43A60. 


\section{Introduction}

In recent years, fractional differential equations have been gaining considerable attention of many scientists and mathematicians because of their demonstrated applications in widespread fields of science and engineering. Since noises or stochastic perturbations are unavoidable and omnipresent in nature as well as in man-made systems, so we have to move from deterministic models to stochastic models. Stochastic differential equations play an important role in formulation and analysis of fluctuations in stock market prices, asset prices, population modeling, control engineering, and chemical engineering [12, 20] ect. Motivated by these facts many researchers are showing great interest to establish an appropriate system to investigate qualitative properties such as existence, uniqueness, controllability and stability of these physical processes with the help of fractional calculus, stochastic analysis and fixed point theorems. For more details, we refer to $[1,3,10,11,16,19,28,29]$ and references therein.

On the other hand, the study of differential equations with impulsive effect constitutes a useful and important field of research due to a lot of applications. In particular, differential equations with impulsive effects arise in various deterministic and stochastic processes which appear in chemical technology, physics, medicine and economics ect. The fractional differential equations involving impulsive effects came out as a natural description of observed phenomena. For more details see $[5,13,14,21,22,24]$ and the references therein.

The concept of pseudo almost periodic solutions introduced by Zhang $[25,26]$ is a natural and good generalization of the classical almost periodic functions. Further, Diagana investigated weighted pseudo almost periodic solutions in [8]. Moreover, the authors investigated piecewise almost periodic solutions in [22], piecewise square mean almost periodic solutions in [11], pseudo almost periodic solutions in [5, 27] for impulsive differential equations. Recently, Zhinan [23] analyzed piecewise weighted pseudo almost periodic functions, which was more tricky and changeable than those of the classical functions. Many authors have been made important contributions in study of almost periodic functions and its generalizations, one can see $[6,11,13,14,22,23,24]$ and the references therein. However, piecewise square mean weighted pseudo almost periodic mild solutions for the fractional order stochastic impulsive differential equations, is an untreated topic in the literature and this fact is the motivation of the present work.

In this paper, we are interested to investigate the existence and uniqueness of piecewise square mean weighted pseudo almost periodic mild solution for the following fractional order stochastic impulsive differential system

$$
\begin{aligned}
{ }^{c} D^{\alpha} y(t)+A y(t) & =G(t, y(t))+F\left(t, y(t), \int_{-\infty}^{t} \mathcal{K}(t-s) g(s, y(s)) d s\right) \frac{d w(t)}{d t}, \quad t_{0}<t \neq t_{i}, t \in \mathbb{R} \\
y\left(t_{i}^{+}\right) & =y\left(t_{i}^{-}\right)+\mathcal{G}_{i}\left(y\left(t_{i}\right)\right), \quad i \in \mathbb{Z} \\
y\left(t_{0}\right) & =y_{0},
\end{aligned}
$$

where the state $y(\cdot)$ take values in $\mathbb{L}^{2}(\mathbb{P}, \mathbb{H}), \mathbb{H}$ is a separable real Hilbert space; ${ }^{c} \mathrm{D}^{\alpha}, \alpha \in(0,1)$ 
symbolizes the Caputo fractional derivative of order $\alpha ;-A: \mathcal{D}(A) \subset \mathbb{L}^{2}(\mathbb{P}, \mathbb{H}) \rightarrow \mathbb{L}^{2}(\mathbb{P}, \mathbb{H})$, is the infinitesimal generator of an analytic semigroup of exponentially bounded linear operator $\{\mathcal{S}(t)\}_{t \geq 0} ;\{w(t): t \geq 0\}$ is a $\mathbb{K}$-valued Wiener process, $\mathbb{K}$ is another separable Hilbert space; $G, F, \mathcal{G}_{i}$ are some suitable functions will be mention later; $\delta(\cdot)$ is Dirac's delta function and $\mathcal{K} \in \mathrm{L}^{1}(\mathbb{R})$ with $|\mathcal{K}(\mathrm{t})| \leq \mathrm{C}_{\mathcal{K}} e^{-\mathrm{bt}}, \mathrm{b}, \mathrm{C}_{\mathcal{K}}>0$.

The rest of this paper is organized as follows: In section 2, we define some fundamental results about the notion of piecewise square mean weighted pseudo almost periodic functions. Section 3 is devoted to the main results ensuring the existence and uniqueness of mild solutions of (1.1) - (1.3) via fractional power of operator and fixed point technique. At last, we will provide an example to show the feasibility of the theory discussed in this paper.

\section{Preliminaries}

Let $\mathcal{L}(\mathbb{K}, \mathbb{H})$ denote the collection of all bounded linear operators form $\mathbb{K}$ to $\mathbb{H}$. For convenience, without confusion we will employ the same notation $\|$.$\| to denote the norms in \mathbb{H}, \mathbb{K}$ and $\mathcal{L}(\mathbb{K}, \mathbb{H})$ and $\langle\cdot, \cdot\rangle$ for inner product in $\mathbb{H}$ and $\mathbb{K}$. Let $\left(\Omega, \mathcal{F},\left\{\mathcal{F}_{t}\right\}_{t} \geq 0, \mathbb{P}\right)$ be a complete probability space equipped with a normal filtration $\left\{\mathcal{F}_{\mathbf{t}}\right\}_{\mathbf{t} \geq 0}$ satisfying the usual conditions(i.e right continuous and $\left\{\mathcal{F}_{0}\right\}$ containing all $\mathbb{P}$-null sets). Suppose $\{w(t): t \geq 0\}$ is a $\mathbb{K}$-valued Wiener process with a finite nuclear covariance operator $\mathrm{Q} \geq 0$ denote $\operatorname{Tr}(\mathrm{Q})=\sum_{\mathrm{k}=1}^{\infty} \widetilde{\lambda}_{k}=\widetilde{\lambda}<\infty$ with $\mathrm{Q} e_{k}=\widetilde{\lambda_{k}} e_{k}$, where

$e_{k}$ are complete orthonormal basis of $\mathbb{K}$. In fact, $w(t)=\sum_{k=1}^{\infty} \sqrt{\widetilde{\lambda}_{k}} w_{k}(t) e_{k}$, here $\left\{w_{k}(t)\right\}_{k=1}^{\infty}$ are mutually independent one dimensional standard Wiener process. We consider that $\mathcal{F}_{\mathrm{t}}=\{w(\mathrm{~s})$ : $0 \leq \mathrm{s} \leq \mathrm{t}\}$ is the $\sigma$ algebra generated by $w$. Assume that $\mathrm{L}_{2}^{0}=\mathrm{L}_{2}\left(\mathrm{Q}^{\frac{1}{2}} \mathbb{K}, \mathbb{H}\right)$ represent the space of all Hilbert Schmidt operators from $Q^{\frac{1}{2}} \mathbb{K}$ to $\mathbb{H}$ with inner product $\langle\phi, \psi\rangle=\operatorname{Tr}\left[\phi Q \psi^{*}\right]$. For more details we refer to the book by Da Prato and Zabczyk [7]. Let the collection of all strongly measurable, square integrable $\mathbb{H}$ valued random variables be denoted by $\mathbb{L}^{2}(\mathbb{P}, \mathbb{H})$ which a Banach space endowed with the norm $\|x(\cdot)\|_{\mathbb{L}^{2}}=\left(\mathbb{E}\|x(\cdot)\|^{2}\right)^{\frac{1}{2}}$, where $\mathbb{E}(\cdot)$ represents the expectations with measure $\mathbb{P}$. Moreover $\mathbb{L}_{\mathcal{F}_{0}}^{2}(\mathbb{P}, \mathbb{H})$ denote the collection of all $\mathcal{F}_{0}$ measurable, $\mathbb{H}$ valued random variable $y(0)$.

Let $\Omega$ be a subspace of $\mathbb{L}^{2}(\mathbb{P}, \mathbb{H})$ and $\mathbb{E}$ be a compact set of $\Omega$. Assume that $\mathbb{R}, \mathbb{N}, \mathbb{Z}$, and $\mathbb{C}$ represent the sets of real number, natural number, integers and complex numbers respectively. For $A$ being a linear operator on $\mathbb{L}^{2}(\mathbb{P}, \mathbb{H}), \mathcal{D}(A), \mathcal{R}(A)$ and $\rho(A)$ stands for domain, range and resolvent of $A$, repectively. Let $\mathcal{B}=\left\{t_{i}: t_{i} \in \mathbb{R}, t_{i}<t_{i+1}, i \in \mathbb{Z}\right\}$ be the set of all strictly increasing and unbounded sequences. For $\left\{t_{i}: i \in \mathbb{Z}\right\} \in \mathcal{B}$, let $\mathcal{P C}\left(\mathbb{R}, \mathbb{L}^{2}(\mathbb{P}, \mathbb{H})\right)$ denote the space of all piecewise stochastically continuous processes $y: \mathbb{R} \rightarrow \mathbb{L}^{2}(\mathbb{P}, \mathbb{H})$ such that $y(t)$ is stochastically continuous at $\mathrm{t}$ for any $\mathrm{t} \notin \mathcal{B}, \mathrm{y}\left(\mathrm{t}_{\mathrm{i}}^{-}\right), \mathrm{y}\left(\mathrm{t}_{\mathrm{i}}^{+}\right)$exists and $\mathrm{y}\left(\mathrm{t}_{\mathrm{i}}^{-}\right)=\mathrm{y}\left(\mathrm{t}_{\mathrm{i}}\right)$ for all $\mathrm{i} \in \mathbb{R}$. In particular, the space $\mathcal{P C}\left(\mathbb{R} \times \Omega, \mathbb{L}^{2}(\mathbb{P}, \mathbb{H})\right)$ consists of all piecewise stochastically continuous processes $y$ : $\mathbb{R} \times \Omega \rightarrow \mathbb{L}^{2}(\mathbb{P}, \mathbb{H})$ such that for any $x \in \Omega, y(t, \cdot) \in \mathcal{P C}\left(\mathbb{R}, \mathbb{L}^{2}(\mathbb{P}, \mathbb{H})\right)$ and for any $t \in \mathbb{R}, y(t, \cdot)$ is stochastically continuous at $x \in \Omega$. 


\subsection{Fractional calculus and fractional power operator}

Following $[16,18])$ we recall some definitions and basic results of fractional calculus.

Definition 1. The Riemann-Liouville fractional integral of a function $\mathrm{g} \in \mathrm{L}_{\mathrm{loc}}^{1}\left(\mathbb{R}^{+}, \mathbb{R}\right)$ with the lower limit zero of order $\alpha>0$ is defined by

$$
J^{\alpha} g(t)=\frac{1}{\Gamma(\alpha)} \int_{0}^{t}(t-\xi)^{\alpha-1} g(\xi) d \xi, \quad t>0,
$$

and $\mathrm{J}^{\mathrm{O}} \mathrm{g}(\mathrm{t}):=\mathrm{g}(\mathrm{t})$. This fractional integral satisfies the properties $\mathrm{J}^{\alpha} \circ \mathrm{J}^{\mathrm{b}}=\mathrm{J}^{\alpha+\mathrm{b}}$ for $\mathrm{b}>0$.

Definition 2. The Riemann-Liouville fractional derivative of a function $\mathrm{g} \in \mathrm{L}_{\mathrm{loc}}^{1}\left(\mathbb{R}^{+}, \mathbb{R}\right)$ with the lower limit zero of order $\alpha>0, \mathrm{n}-1<\alpha<\mathrm{n}, \mathrm{n} \in \mathbb{N}$ is given by

$$
D^{\alpha} g(t)=\frac{1}{\Gamma(n-\alpha)} \frac{d^{n}}{d t^{n}} \int_{0}^{t}(t-\xi)^{n-\alpha-1} g(\xi) d \xi,
$$

Moreover $\mathrm{D}^{0} \mathrm{~g}(\mathrm{t})=\mathrm{g}(\mathrm{t})$ and $\mathrm{D}^{\alpha} \mathrm{J}^{\alpha} \mathrm{g}(\mathrm{t})=\mathrm{g}(\mathrm{t})$ for $\mathrm{t}>0$.

Definition 3. The Caputo fractional derivative of a function $\mathrm{g}:[0, \infty) \rightarrow \mathbb{R}$ with the lower limit 0 of order $\alpha>0$ is given by

$$
{ }^{c} D^{\alpha} g(t)=D^{\alpha}\left(g(t)-\sum_{k=0}^{n-1} \frac{t^{k}}{k !} g^{(k)}(0)\right), \quad t>0, n-1<\alpha<n .
$$

Remark 1. (i) If $\mathrm{g}(\mathrm{t}) \in \mathcal{C}^{\mathrm{n}}([0, \infty))$, then

$$
{ }^{c} D^{\alpha} g(t)=\frac{1}{\Gamma(n-\alpha)} \int_{0}^{t}(t-\xi)^{n-\alpha-1} \frac{d^{n}}{d \xi^{n}} g(\xi) d \xi,
$$

where $\mathrm{n}-1<\alpha<\mathrm{n}, \mathrm{n} \in \mathbb{N}$.

(ii) If $\mathrm{g}$ is an abstract function with values in $\mathbb{H}$, then integral defined in Definition 1 and 2 are taken in Bochner's sense.

If $-A$ generates an analytic semigroup $\mathcal{S}(t)$ in $L^{2}(\mathbb{P}, \mathbb{H})$ and $0 \in \rho(A)$, then for $\sigma>0$, we can define fractional power $A^{-\sigma}$ of the operator $A$ by

$$
A^{-\sigma}=\frac{1}{\Gamma(\sigma)} \int_{0}^{\infty} t^{\sigma-1} \mathcal{S}(t) d t
$$

where $A^{-\sigma}$ is bijective, bounded and $A^{\sigma}=\left(A^{-\sigma}\right)^{-1}, \sigma>0$ a closed linear operator on $\mathcal{D}\left(A^{\sigma}\right)$ such that $\mathcal{D}\left(A^{\sigma}\right)=\mathcal{R}\left(A^{-\sigma}\right)$. Moreover $\mathcal{D}\left(A^{\sigma}\right)$ is dense in $\mathrm{L}^{2}(\mathbb{P}, \mathbb{H})$ and the expression $\|y\|_{\sigma}=\left\|A^{\sigma} y\right\|, y \in \mathcal{D}\left(A^{\sigma}\right)$ defines a norm on $\mathcal{D}\left(A^{\sigma}\right)$. Let us denote by $L^{2}\left(P, \mathbb{H}_{\sigma}\right)$ the Banach space $\mathcal{D}\left(A^{\sigma}\right)$ with norm $\|\cdot\|_{\sigma}$. The following properties are well recognized.

Lemma 2.1. [17] Let $\mathrm{A}$ be an infinitesimal generator of an analytic semigroup $\mathcal{S}(\mathrm{t})$ and $0 \in \rho(A)$. Then 
(i) $\mathcal{S}(\mathrm{t}): \mathrm{L}^{2}(\mathbb{P}, \mathbb{H}) \rightarrow \mathcal{D}\left(\mathrm{A}^{\sigma}\right)$, for $\sigma \geq 0$, and $\mathrm{t}>0$.

(ii) For every $\mathrm{y} \in \mathcal{D}\left(\mathrm{A}^{\sigma}\right)$, we have $\mathcal{S}(\mathrm{t}) \mathrm{A}^{\sigma} \mathrm{y}=\mathrm{A}^{\sigma} \mathcal{S}(\mathrm{t}) \mathrm{y}$.

(iii) The operator $A^{\sigma} \mathcal{S}(\mathrm{t})$ is bounded and

$$
\left\|A^{\sigma} \mathcal{S}(\mathrm{t})\right\| \leq M_{\sigma} t^{-\sigma} e^{-\lambda t}, \quad M_{\sigma}, t, \lambda>0
$$

(iv) For $\mathrm{y} \in \mathcal{D}\left(A^{\sigma}\right)$, and $0<\sigma \leq 1$, we have

$$
\|\mathcal{S}(t) y-y\| \leq C_{\sigma} t^{\sigma}\left\|A^{\sigma} y\right\|, \quad C_{\sigma}>0 .
$$

\subsection{Square-mean piecewise weighted pseudo almost periodic function}

Now we define square-mean piecewise weighted pseudo almost periodic function and explore its properties

Definition 4. A stochastic process $y: \mathbb{R} \rightarrow \mathbb{L}^{2}(\mathbb{P}, \mathbb{H})$ is said to be stochastically continuous for $\mathrm{s} \in \mathbb{R}$ if $\lim _{\mathrm{t} \rightarrow \mathrm{s}} \mathbb{E}\|\mathrm{y}(\mathrm{t})-\mathrm{y}(\mathrm{s})\|^{2}=0$.

Definition 5. A stochastically continuous process $y: \mathbb{R} \rightarrow \mathbb{L}^{2}(\mathbb{P}, \mathbb{H})$ is said to be square mean almost periodic if for ever $\epsilon>0$, there exists a $\mathrm{l}(\epsilon)>0$ such that every interval $\mathrm{L}$ of length $l(\epsilon)>0$ contains a number $\tau$ with the property $\mathbb{E}\|\mathrm{y}(\mathrm{t}+\tau)-\mathrm{y}(\mathrm{t})\|^{2}<\epsilon$ for all $\mathrm{t} \in \mathbb{R}$.

Definition 6. A sequence $z_{i}: \mathbb{Z} \rightarrow \mathbb{L}^{2}(\mathbb{P}, \mathbb{H})$ is said to be square-mean almost periodic sequence if for ever $\epsilon>0$, there exists a $l(\epsilon)>0$ such that every $p \in \mathbb{Z}$ there is at least one number $\mathrm{k}$ in $[p, p+l]$, with the property $\mathbb{E}\left\|z_{i+k}-z_{i}\right\|^{2}<\epsilon$ for all $i \in \mathbb{Z}$. We denote the set of all such processes by $\mathcal{A P}\left(\mathbb{Z}, \mathbb{L}^{2}(\mathbb{P}, \mathbb{H})\right)$.

Remark 2. Let $\left\{z_{i}\right\} \in \mathcal{A P}\left(\mathbb{Z}, \mathbb{L}^{2}(\mathbb{P}, \mathbb{H})\right)$, then $\left\{z_{i}: i \in \mathbb{Z}\right\}$ is stochastically bounded.

Let $W_{d}$ denote the collection of all functions (weights) $\rho_{m}: \mathbb{Z} \rightarrow(0,+\infty), m \in \mathbb{Z}$. For $\rho_{\mathrm{m}} \in W_{\mathrm{d}}$ and $\mathrm{m} \in \mathbb{Z}^{+}=\{\mathrm{m} \in \mathbb{Z}, \mathrm{m} \geq 0\}$, set $\mu(\mathrm{m}, \rho):=\sum_{k=-m}^{\mathrm{m}} \rho_{\mathrm{m}}$. Denote $W_{\mathrm{d}, \infty}:=\left\{\rho \in W_{\mathrm{d}}\right.$ : $\left.\lim _{m \rightarrow \infty}(m, \rho)=\infty\right\}$.

For $\rho \in W_{\mathrm{d}, \infty}$, we define

$$
\mathcal{P} \mathcal{A} \mathcal{P}_{\rho}\left(\mathbb{Z}, \mathbb{L}^{2}(\mathbb{P}, \mathbb{H})\right)=\left\{z_{\mathrm{m}} \in l^{\infty}\left(\mathbb{Z}, \mathbb{L}^{2}(\mathbb{P}, \mathbb{H})\right): \lim _{\mathrm{m} \rightarrow \infty} \frac{1}{\mu(\mathrm{m}, \rho)} \sum_{k=-m}^{m} \mathbb{E}\left\|z_{\mathrm{m}}\right\|^{2} \rho_{\mathrm{m}}=0\right\}
$$

Definition 7. Let $\rho \in W_{d, \infty}$. A sequence $\left\{z_{i}\right\}_{i \in \mathbb{Z}} \in l^{\infty}\left(\mathbb{Z}, \mathbb{L}^{2}(\mathbb{P}, \mathbb{H})\right)$ is called square mean discrete weighted pseudo almost periodic if $z_{i}=a_{i}+b_{i}$, where $a_{i} \in \mathcal{A P}\left(\mathbb{Z}, \mathbb{L}^{2}(\mathbb{P}, \mathbb{H})\right)$ and $b_{i} \in$ $\mathcal{P} \mathcal{A} \mathcal{P}_{\rho}\left(\mathbb{Z}, \mathbb{L}^{2}(\mathbb{P}, \mathbb{H})\right)$. The set of all such functions denoted by $\mathcal{W P} \mathcal{A} \mathcal{P}_{\rho}\left(\mathbb{Z}, \mathbb{L}^{2}(\mathbb{P}, \mathbb{H})\right)$.

Definition 8. A stochastic process $y \in \mathcal{P C}\left(\mathbb{R}, \mathbb{L}^{2}(\mathbb{P}, \mathbb{H})\right)$ is said to be square-mean piecewise almost periodic if: 
(i) The set of all sequences $\left\{t_{i}^{j}: t_{i}^{j}:=t_{i+j}-t_{i}, t_{i} \in \mathcal{B}, i, j \in \mathbb{Z}\right\}$ are equipotentially almost periodic i.e. for every $\epsilon>0$ there exists a relatively $\mathrm{D}_{\epsilon} \subset \mathbb{R}$ of $\epsilon$ periods common for all sequences $\left\{t_{i}^{j}\right\}$.

(ii) For any $\epsilon>0$, there exists a $\delta>0$ such that if the points $\mathrm{s}$ and $\mathrm{t}$ are in the same interval of continuity of $\mathrm{y}(\mathrm{t})$ and $|\mathrm{t}-\mathrm{s}|<\delta$, then $\mathbb{E}\|\mathrm{y}(\mathrm{t})-\mathrm{y}(\mathrm{s})\|^{2}<\epsilon$.

(iii) For any $\epsilon>0$, there exists a relatively dense set $\mathrm{R}_{\epsilon}$ of $\mathbb{R}$ such that if $\tau \in \mathrm{R}_{\epsilon}$, then $\mathbb{E} \| \mathrm{y}(\mathrm{t}+$ $\tau)-\mathrm{y}(\mathrm{t}) \|^{2}<\epsilon$, with the condition $\left|\mathrm{t}-\mathrm{t}_{\mathrm{i}}\right|>\epsilon, \mathrm{i} \in \mathbb{Z}$.

We denote by $\mathcal{A P}^{\mathfrak{p}}\left(\mathbb{R}, \mathbb{L}^{2}(\mathbb{P}, \mathbb{H})\right)$ the space of all square-mean piecewise almost periodic processes. We denote by $\mathcal{U P C}\left(\mathbb{R}, \mathbb{L}^{2}(\mathbb{P}, \mathbb{H})\right)$ the space of all stochastic processes such that $y$ satisfy the condition (ii) in Definition 8 and $y \in \mathcal{P C}\left(\mathbb{R}, \mathbb{L}^{2}(\mathbb{P}, \mathbb{H})\right)$.

Definition 9. [6] For $\left\{\mathrm{t}_{i}\right\} \in \mathcal{B}, i \in \mathbb{Z}$, the function $\mathrm{f}(\mathrm{t}, \mathrm{y}) \in \mathcal{P C}\left(\mathbb{R} \times \Omega, \mathbb{L}^{2}(\mathbb{P}, \mathbb{H})\right)$ is called squaremean piecewise almost periodic in $\mathrm{t} \in \mathbb{R}$ and uniformly on $\mathbb{E} \subseteq \Omega,\{\mathrm{f}(\cdot, \mathrm{y}): \mathrm{y} \in \mathbb{E}\}$ is uniformly bounded, and for every $\epsilon>0$ there exists a relatively compact set $R_{\epsilon}$ of $\mathbb{R}$, such that $\mathbb{E} \| f(t+\tau, y)-$ $\mathrm{f}(\mathrm{t}, \mathrm{y}) \|^{2}<\epsilon$, for all $\mathrm{y} \in \mathbb{E}, \mathrm{t} \in \mathbb{R}$ and $\tau \in \mathrm{R}_{\epsilon}$ with $\left|\mathrm{t}-\mathrm{t}_{\mathrm{i}}\right|>\epsilon, \boldsymbol{i} \in \mathbb{Z}$. The set of all such processes is denoted by $\mathcal{A P}^{\mathrm{p}}\left(\mathbb{R} \times \Omega, \mathbb{L}^{2}(\mathbb{P}, \mathbb{H})\right)$.

Lemma 2.2. [13] Let $\mathrm{f} \in \mathcal{A P}^{\mathfrak{p}}\left(\mathbb{R}, \mathbb{L}^{2}(\mathbb{P}, \mathbb{H})\right),\left\{z_{i}: i \in \mathbb{Z}\right\}$ is square mean almost periodic sequence in $\mathbb{L}^{2}(\mathbb{P}, \mathbb{H})$ and $\left\{t_{i}^{j}: i, j \in \mathbb{Z}\right\}$ is equipotentially almost periodic. Then for each $\epsilon>0$ there exist relatively dense sets $\mathbb{R}_{\epsilon}$ of $\mathbb{R}$ and $\mathbf{Z}_{\epsilon}$ of $\mathbb{Z}$ such that the following conditions hold:

(i) $\mathbb{E}\|f(t+\tau)-f(t)\|^{2}<\epsilon$ for all $\tau \in R_{\epsilon}, t \in \mathbb{R},\left|t-t_{i}\right|>\epsilon, i \in \mathbb{Z}$.

(ii) $\mathbb{E}\left\|z_{i+p}-z_{\mathfrak{i}}\right\|^{2}<\epsilon$ for all $p \in Z_{\epsilon}$, and $i \in \mathbb{Z}$.

(iii) For any $\tau \in R_{\epsilon}$ there exists at least a number $p \in Z_{\epsilon}$ such that $\left|\mathrm{t}_{i}^{\mathrm{p}}-\tau\right|<\epsilon, \mathfrak{i} \in \mathbb{Z}$.

Next, we introduce the concept of piecewise square mean weighted pseudo almost periodic functions and explore its properties.

Let $W$ be the collections of all positive and locally integrable functions $\rho: \mathbb{R} \rightarrow(0, \infty)$. For each $\rho \in W$ and $\gamma>0$, set $\mu(\gamma, \rho):=\int_{-\gamma}^{\gamma} \rho(t) d t$.

Define

$$
\begin{array}{r}
W_{\infty}:=\left\{\rho \in W: \lim _{\gamma \rightarrow \infty} \mu(\gamma, \rho)=\infty\right\}, \\
W_{B}:=\left\{\rho \in W_{\infty}: \rho \text { is bounded and } \inf _{t \in \mathbb{R}} \rho(t)>0\right\} .
\end{array}
$$

It is clear that $W_{B} \subset W_{\infty} \subset W$.

Definition 10. Let $\rho_{1}, \rho_{2} \in W_{\infty}$. $\rho_{1}$ is said to be equivalent to $\rho_{2}$ (i.e. $\rho_{1} \sim \rho_{2}$ ) if $\frac{\rho_{1}}{\rho_{2}} \in W_{B}$. 
It is clear that " $\sim$ " binary equivalence relation on $W_{\infty}$. For a given weight $\rho \in W_{\infty}$, the equivalence class is denoted by $C_{L}(\rho):=\left\{\rho^{*} \in W_{\infty}: \rho \sim \rho^{*}\right\}$. Moreover $W_{\infty}=\cup_{\rho \in W_{\infty}} C_{L}(\rho)$. For $\rho \in W_{\infty}$, we define

$$
\mathcal{P} \mathcal{A} \mathcal{A}_{\rho}\left(\mathbb{R}, \mathbb{L}^{2}(\mathbb{P}, \mathbb{H})\right):=\left\{f \in \mathcal{P C}\left(\mathbb{R}, \mathbb{L}^{2}(\mathbb{P}, \mathbb{H})\right): \lim _{\gamma \rightarrow \infty} \frac{1}{\mu(\gamma, \rho)} \int_{-\gamma}^{\gamma} \mathbb{E}\|f(t)\|^{2} \rho(t) d t=0\right\} .
$$

Similarly

$$
\begin{aligned}
& \mathcal{P A \mathcal { A }}_{\rho}\left(\mathbb{R} \times \Omega, \mathbb{L}^{2}(\mathbb{P}, \mathbb{H})\right) \\
& :=\left\{\mathrm{f} \in \mathcal{P C}\left(\mathbb{R} \times \Omega, \mathbb{L}^{2}(\mathbb{P}, \mathbb{H})\right): \lim _{\gamma \rightarrow \infty} \frac{1}{\mu(\gamma, \rho)} \int_{-\gamma}^{\gamma} \mathbb{E}\|\mathrm{f}(\mathrm{t}, \mathrm{y})\|^{2} \rho(\mathrm{t}) \mathrm{dt}=0 \text { uniformly in } \mathrm{y} \in \mathbb{E}\right\} .
\end{aligned}
$$

Definition 11. A function $\mathrm{f} \in \mathcal{P C}\left(\mathbb{R}, \mathbb{L}^{2}(\mathbb{P}, \mathbb{H})\right)$ is called piecewise square mean weighted pseudo almost periodic if it has a decomposition of the form $\mathrm{f}=\phi+\psi$, where $\phi \in \mathcal{A P}^{\mathrm{p}}\left(\mathbb{R}, \mathbb{L}^{2}(\mathbb{P}, \mathbb{H})\right)$ and $\psi \in \mathcal{P} \mathcal{A} \mathcal{P}_{\rho}\left(\mathbb{R}, \mathbb{L}^{2}(\mathbb{P}, \mathbb{H})\right)$. The set of all such functions denoted by $\mathcal{W P} \mathcal{A} \mathcal{P}_{\rho}\left(\mathbb{R}, \mathbb{L}^{2}(\mathbb{P}, \mathbb{H})\right)$.

Definition 12. A function $f \in \mathcal{P C}\left(\mathbb{R} \times \Omega, \mathbb{L}^{2}(\mathbb{P}, \mathbb{H})\right)$ is called piecewise square mean weighted pseudo almost periodic if it has a decomposition of the form $\mathrm{f}=\phi+\psi$, where $\phi \in \mathcal{A P}^{\mathfrak{p}}(\mathbb{R} \times$ $\left.\Omega, \mathbb{L}^{2}(\mathbb{P}, \mathbb{H})\right)$ and $\psi \in \mathcal{P} \mathcal{A} \mathcal{P} \rho\left(\mathbb{R} \times \Omega, \mathbb{L}^{2}(\mathbb{P}, \mathbb{H})\right)$. The set of all such functions denoted by $\mathcal{W P} \mathcal{A} \mathcal{P}_{\rho}(\mathbb{R} \times$ $\left.\Omega, \mathbb{L}^{2}(\mathbb{P}, \mathbb{H})\right)$.

For $\rho \in W_{\infty}$ and $\tau \in \mathbb{R}$ define $\rho^{\tau}$ by $\rho^{\tau}(t)=\rho(t+\tau)$ for all $t \in \mathbb{R}$. Define

$$
W_{T}=\left\{\rho \in W_{\infty}: \rho \sim \rho^{\tau} \text { for each } t \in \mathbb{R}\right\} .
$$

It is clear that $W_{T}$ contains many of weights, such as $1, e^{t}$ and $1+|t|^{n}$ with $n \in \mathbb{N}$ among others.

Remark 3. (i) For $\rho \in W_{\mathrm{T}}, \mathcal{P} \mathcal{A} \mathcal{P}\left(\mathbb{R}, \mathbb{L}^{2}(\mathbb{P}, \mathbb{H})\right)$ is a translation invariant set of $\mathcal{P C}\left(\mathbb{R}, \mathbb{L}^{2}(\mathbb{P}, \mathbb{H})\right)$.

(ii) It is easy to see that $\mathcal{W} \mathcal{P} \mathcal{A} \mathcal{P}_{\rho}\left(\mathbb{R}, \mathbb{L}^{2}(\mathbb{P}, \mathbb{H})\right)\left(\right.$ resp., $\left.\mathcal{W} \mathcal{P} \mathcal{A} \mathcal{P}_{\rho}\left(\mathbb{R} \times \Omega, \mathbb{L}^{2}(\mathbb{P}, \mathbb{H})\right)\right)$ are Banach spaces with sup norm.

Similar as the proof of Lemma 2.5 in [9], we have the following result.

Lemma 2.3. Let $\left\{f_{n}\right\}_{n \in \mathbb{N}}$ be a sequence of functions in $\mathcal{W P}_{\mathcal{P}} \mathcal{P} \rho\left(\mathbb{R}, \mathbb{L}^{2}(\mathbb{P}, \mathbb{H})\right)$. If $\mathrm{f}_{\mathrm{n}}$ converge uniformly to $\mathrm{f}$, then $\mathrm{f} \in \mathcal{W} \mathcal{P} \mathcal{A} \mathcal{P}_{\rho}\left(\mathbb{R}, \mathbb{L}^{2}(\mathbb{P}, \mathbb{H})\right)$.

Similar as the proof of [14] the following composition theorems hold for piecewise square mean weighted pseudo almost periodic functions.

Theorem 2.1. Let $\mathrm{f}(\mathrm{t}, \mathrm{y}, z) \in \mathcal{W} \mathcal{P} \mathcal{A} \mathcal{P}_{\rho}\left(\mathbb{R} \times \Omega \times \Omega, \mathbb{L}^{2}(\mathbb{P}, \mathbb{H})\right), \xi, \chi \in \mathcal{W P} \mathcal{A} \mathcal{P}_{\rho}\left(\mathbb{R}, \mathbb{L}^{2}(\mathbb{P}, \mathbb{H})\right)$ and $\mathcal{R}(\xi) \times \mathcal{R}(\xi) \subset \Omega \times \Omega$. Assume that there exists a number $\mathrm{L}_{\mathrm{f}}>0$ such that

$\mathbb{E}\left\|\mathbf{f}\left(t, y_{1}, z_{1}\right)-f\left(t, y_{2}, z_{2}\right)\right\|^{2} \leq L_{f} .\left(\mathbb{E}\left\|y_{1}-y_{2}\right\|^{2}+\mathbb{E}\left\|z_{1}-z_{2}\right\|^{2}\right), \quad$ for all $t \in \mathbb{R}, y_{i}, z_{i} \in \Omega, i=1,2$, then $f(\cdot, \xi(\cdot), \chi(\cdot)) \in \mathcal{W} \mathcal{P} \mathcal{A} \mathcal{P}_{\rho}\left(\mathbb{R}, \mathbb{L}^{2}(\mathbb{P}, \mathbb{H})\right)$. 
Theorem 2.2. Let $\left\{I_{i}(y): i \in \mathbb{Z}\right\}$ for any $y \in \Omega$ be a piecewise square mean weighted pseudo almost periodic sequence. Assuming that there exists a constant $\mathrm{L}_{0}>0$ such that

$$
\mathbb{E}\left\|I_{i}(x)-I_{i}(y)\right\|^{2} \leq L_{0} \cdot \mathbb{E}\|x-y\|^{2}, \text { for all } x, y \in \Omega, i \in \mathbb{Z} .
$$

If $\xi \in \mathcal{W P} \mathcal{A} \mathcal{P} \rho\left(\mathbb{R}, \mathbb{L}^{2}(\mathbb{P}, \mathbb{H})\right) \cap \mathcal{U P C}\left(\mathbb{R}, \mathbb{L}^{2}(\mathbb{P}, \mathbb{H})\right)$ such that $\mathcal{R}(\xi) \subset \Omega$, then $\mathrm{I}_{i}\left(\xi\left(\mathrm{t}_{i}\right)\right)$ is piecewise square mean weighted pseudo almost periodic.

Lemma 2.4. [21] Assume that $\left\{t_{i}^{j}: i, j \in \mathbb{Z}\right\}$ are equipotentially almost periodic sequences, then for each $\mathrm{p}>0$ there exists a positive integer $\mathrm{N}_{0}$ such that each interval of length $\mathrm{p}$ has no more than $\mathrm{N}_{\mathrm{o}}$ elements of the sequence $\left\{\mathrm{t}_{\mathrm{i}}\right\}$ and

$$
n(s, t) \leq N_{0}(t-s)+N_{0}
$$

where $\mathrm{n}(\mathrm{t}, \mathrm{s})$ denotes the number of the points $\mathrm{t}_{\mathrm{i}}$ in the interval $[\mathrm{t}, \mathrm{s}]$.

\section{Main Results}

In this section, we establish piecewise square mean weighted pseudo almost periodic mild solution to the fractional order stochastic impulsive differential system (1.1)-(1.3).

In formulation of the system (1.1)-(1.3), we consider the following assumptions:

$\left(H_{1}\right)$ The collection of sequences $\left\{t_{i}^{j}: i, j \in \mathbb{Z}\right\}$ is equipotentially almost periodic and there exists $\theta>0$ such that $\inf _{i} \tau_{i}^{1}=\theta$.

$\left(\mathrm{H}_{2}\right)-\mathrm{A}$ is the infinitesimal generator of an analytic semigroup $\mathcal{S}(\mathrm{t}), \mathrm{t} \geq 0$, on $\mathbb{L}^{2}(\mathbb{P}, \mathbb{H})$.

$\left(\mathrm{H}_{3}\right)$ For $\rho \in W_{\mathrm{T}}, g \in \mathcal{W} \mathcal{P} \mathcal{A} \mathcal{P}_{\rho}\left(\mathbb{R} \times \mathbb{L}^{2}\left(\mathbb{P}, \mathbb{H}_{\sigma}\right), \mathbb{L}^{2}(\mathbb{P}, \mathbb{H})\right)$ and there exists a $\mathrm{L}_{g}>0,0<\eta<1$, such that

$$
\mathbb{E}\left\|g\left(t_{1}, u_{1}\right)-g\left(t_{2}, u_{2}\right)\right\|^{2} \leq L_{g}\left(\left|t_{1}-t_{2}\right|^{\eta}+\mathbb{E}\left\|u_{1}-u_{2}\right\|_{\sigma}^{2}\right),
$$

for each $\left(t_{i}, u_{i}\right) \in \mathbb{R} \times \mathbb{L}^{2}\left(\mathbb{P}, \mathbb{H}_{\sigma}\right) i=1,2$.

$\left(\mathrm{H}_{4}\right)$ For $\rho \in W_{\mathrm{T}}, \mathrm{G} \in \mathcal{W} \mathcal{P} \mathcal{A} \mathcal{P}_{\rho}\left(\mathbb{R} \times \mathbb{L}^{2}\left(\mathbb{P}, \mathbb{H}_{\sigma}\right), \mathbb{L}^{2}(\mathbb{P}, \mathbb{H})\right)$ and there exists $\mathrm{L}_{\mathrm{F}}>0,0<\eta<1$, such that

$$
\mathbb{E}\left\|G\left(t_{1}, u_{1}\right)-G\left(t_{2}, u_{2}\right)\right\|^{2} \leq L_{G}\left(\left|t_{1}-t_{2}\right|^{\eta}+\mathbb{E}\left\|u_{1}-u_{2}\right\|_{\sigma}^{2}\right),
$$

for each $\left(t_{i}, u_{i}\right) \in \mathbb{R} \times \mathbb{L}^{2}\left(\mathbb{P}, \mathbb{H}_{\sigma}\right), i=1,2$.

$\left(\mathrm{H}_{5}\right)$ For $\rho \in W_{\mathrm{T}}, \mathrm{F} \in \mathcal{W} \mathcal{P} \mathcal{A} \mathcal{P}_{\rho}\left(\mathbb{R} \times \mathbb{L}^{2}\left(\mathbb{P}, \mathbb{H}_{\sigma}\right) \times \mathbb{L}^{2}\left(\mathbb{P}, \mathbb{H}_{\sigma}\right), \mathbb{L}^{2}\left(\mathrm{P}, \mathrm{L}_{2}^{0}\right)\right)$ and there exists $\mathrm{L}_{\mathrm{F}}>0,0<$ $\eta<1$, such that

$$
\mathbb{E}\left\|\mathrm{F}\left(\mathrm{t}, \mathrm{u}_{1}, v_{1}\right)-\mathrm{F}\left(\mathrm{t}, \mathrm{u}_{2}, \mathrm{u}_{2}\right)\right\|^{2} \leq \mathrm{L}_{\mathrm{F}}\left(\left|\mathrm{t}_{1}-\mathrm{t}_{2}\right|^{\mathfrak{\eta}}+\mathbb{E}\left\|\mathrm{u}_{1}-\mathrm{u}_{2}\right\|_{\sigma}^{2}+\mathbb{E}\left\|v_{1}-v_{2}\right\|_{\sigma}^{2}\right),
$$

for each $\left(t_{i}, u_{i}, v_{i}\right) \in \mathbb{R} \times \mathbb{L}^{2}\left(\mathbb{P}, \mathbb{H}_{\sigma}\right) \times \mathbb{L}^{2}\left(\mathbb{P}, \mathbb{H}_{\sigma}\right) i=1,2$. 
$\left(\mathrm{H}_{6}\right)\left\{\mathcal{G}_{\mathfrak{i}}(\mathrm{y}): k \in \mathbb{Z}\right\}$ is piecewise square mean weighted pseudo almost periodic sequence uniformly $y \in \Omega$ and that there exists a constant $\mathrm{L}_{\mathcal{G}}>0$ such that

$$
\mathbb{E}\left\|\mathcal{G}_{\mathfrak{i}}(x)-\mathcal{G}_{\mathfrak{i}}(y)\right\|^{2} \leq \mathrm{L}_{\mathcal{G}} \cdot \mathbb{E}\|x-y\|_{\sigma}^{2}, \text { for all } x, y \in \mathbb{L}^{2}\left(\mathbb{P}, \mathbb{H}_{\sigma}\right) .
$$

$\left(\mathrm{H}_{7}\right)$ For any $\mathrm{L}_{1}, \mathrm{~L}_{2}>0$, denote

$\mathrm{C}_{\mathrm{F}}:=\sup _{\mathrm{t} \in \mathbb{R},\|\mathrm{u}\|_{\infty}<\mathrm{L}_{1},\|v\|_{\infty}<\mathrm{L}_{2}}\left(\mathbb{E}\|\mathrm{F}(\mathrm{t}, \mathrm{u}, v)\|^{2}\right)^{\frac{1}{2}}<\infty$, $\mathrm{C}_{\mathrm{G}}:=\sup _{\mathrm{t} \in \mathbb{R},\|\mathrm{u}\|_{\infty}<\mathrm{L}_{1}}\left(\mathbb{E}\|\mathrm{G}(\mathrm{t}, \mathrm{u})\|^{2}\right)^{\frac{1}{2}}<\infty, \mathrm{C}_{\mathcal{G}_{i}}:=\sup _{\mathrm{k} \in \mathbb{Z},\|\mathrm{u}\|_{\infty}<\mathrm{L}_{1}}\left(\mathbb{E}\left\|\mathcal{G}_{\mathrm{i}}(\mathrm{u})\right\|^{2}\right)^{\frac{1}{2}}<\infty$.

Then there exists a constant $r_{0}>0$ such that

$$
3 M_{\sigma}^{2}\left[4 C_{\mathcal{G}_{i}}^{2} N_{0}^{2}\left(\frac{1}{M_{0}^{\sigma}}+\frac{1}{e^{\lambda}-1}\right)^{2}+C_{G}^{2} \frac{\Gamma^{2}(1-\sigma)}{\lambda^{2(1-\sigma)}}+C_{F}^{2} \mathcal{N}_{0} \frac{\Gamma(1-2 \sigma)}{\lambda^{(2-2 \sigma)}}\right] \leq r_{0} .
$$

Now, we define the mild solutions for the system (1.1) - (1.3).

Definition 13. A stochastic process $\mathrm{y} \in \mathcal{P C}\left(\mathrm{J}, \mathrm{L}^{2}(\mathbb{P}, \mathbb{H})\right), \mathrm{J} \subset \mathbb{R}$ is a mild solution of the system (1.1) -(1.3), if

(i) yo $_{0} \in \mathrm{L}_{\mathcal{F}_{0}}^{2}(\mathbb{P}, \mathbb{H})$.

(ii) $\mathrm{y}(\mathrm{t}) \in \mathrm{L}^{2}(\mathbb{P}, \mathbb{H})$ has càdlàg path on $\mathrm{t} \in \mathrm{J}$ a.s., and satisfies the following integral equation

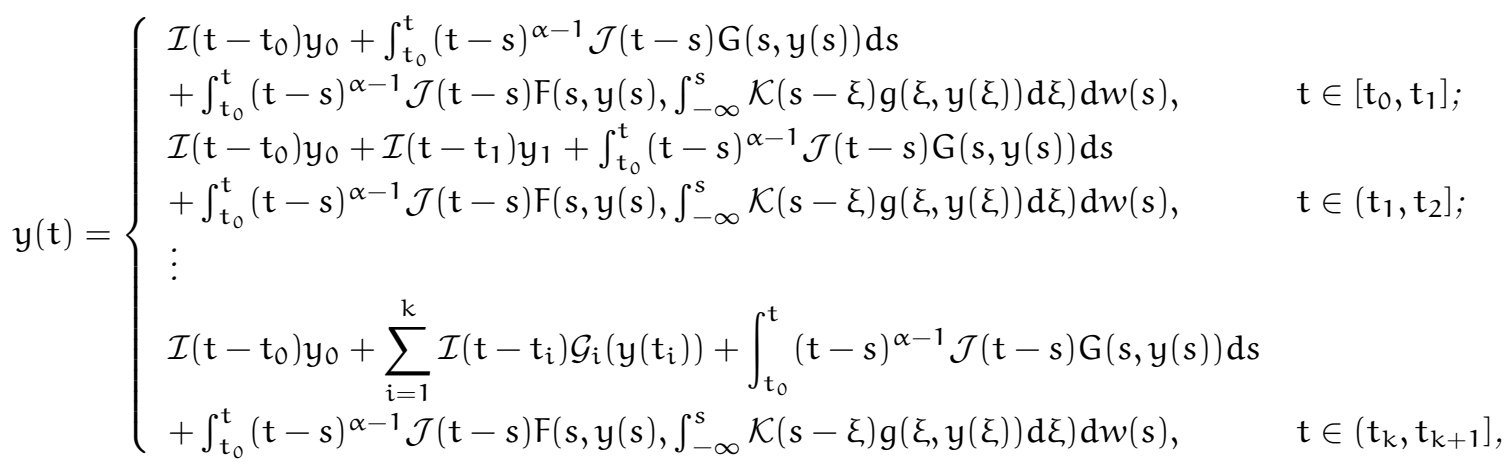

where

$$
\mathcal{I}(\mathrm{t})=\int_{0}^{\infty} \mathcal{N}_{\alpha}(\theta) \mathcal{S}\left(\mathrm{t}^{\alpha} \theta\right) \mathrm{d} \theta, \quad \mathcal{J}(\mathrm{t})=\alpha \int_{0}^{\infty} \theta \mathcal{N}_{\alpha}(\theta) \mathcal{S}\left(\mathrm{t}^{\alpha} \theta\right) \mathrm{d} \theta
$$

and for $\theta \in(0, \infty)$

$$
\mathcal{N}_{\alpha}(\theta)=\frac{1}{\alpha} \theta^{-1-\frac{1}{\alpha}} \omega_{\alpha}\left(\theta^{-\frac{1}{\alpha}}\right) \geq 0, \quad \omega_{\alpha}(\theta)=\frac{1}{\pi} \sum_{n=1}^{\infty}(-1)^{n-1} \theta^{-n \alpha-1} \frac{\Gamma(n \alpha+1)}{n !} \sin (n \pi \alpha),
$$


$\mathcal{N}_{\alpha}$ denote the probability density function on $(0, \infty)$ such that

$$
\mathcal{N}_{\alpha}(\theta) \geq 0, \quad \theta \in(0, \infty) \text { and } \int_{0}^{\infty} \mathcal{N}_{\alpha}(\theta) \mathrm{d} \theta=1
$$

Noth that when $\left(\mathrm{H}_{2}\right)$ holds, we observe that if $y(t)$ is stochastically bounded solution of the system (1.1) - (1.3) on $\mathbb{R}$, then the mild solution (3.1) take the following form as $t_{0} \rightarrow-\infty$.

$$
\begin{aligned}
y(t)= & \sum_{t_{i}<t} \mathcal{I}\left(t-t_{i}\right) \mathcal{G}_{i}\left(y\left(t_{i}\right)\right)+\int_{-\infty}^{t}(t-s)^{\alpha-1} \mathcal{J}(t-s) G(s, y(s)) d s \\
& +\int_{-\infty}^{t}(t-s)^{\alpha-1} \mathcal{J}(t-s) F\left(s, y(s), \int_{-\infty}^{s} \mathcal{K}(s-\xi) g(\xi, y(\xi)) d \xi\right) d w(s) .
\end{aligned}
$$

Lemma 3.1. Assume that $\left(\mathrm{H}_{1}\right)-\left(\mathrm{H}_{3}\right)$ hold, if $\mathrm{y} \in \mathcal{W} \mathcal{P} \mathcal{A} \mathcal{P}_{\rho}\left(\mathbb{R}, \mathbb{L}^{2}\left(\mathbb{P}, \mathbb{H}_{\sigma}\right)\right)$, then

$$
\Theta\left(A^{-\sigma} y\right)(t):=\int_{-\infty}^{t} \mathcal{K}(t-s) g\left(s, A^{-\sigma} y(s)\right) d s \in \mathcal{W P} \mathcal{A} \mathcal{P}_{\rho}\left(\mathbb{R}, \mathbb{L}^{2}(\mathbb{P}, \mathbb{H})\right)
$$

Proof. Since $A^{-\sigma}$ is bounded, $\psi:=g\left(\cdot, A^{-\sigma} y(\cdot)\right) \in \mathcal{W P} \mathcal{A} \mathcal{P}_{\rho}\left(\mathbb{R}, \mathbb{L}^{2}(\mathbb{P}, \mathbb{H})\right)$ by Theorem 2.1. Assume that $\psi=\psi_{1}+\psi_{2}$ with $\psi_{1} \in \mathcal{A P}^{p}\left(\mathbb{R}, \mathbb{L}^{2}(\mathbb{P}, \mathbb{H})\right)$ and $\psi_{2} \in \mathcal{P} \mathcal{A} \mathcal{P}_{\rho}\left(\mathbb{R}, \mathbb{L}^{2}(\mathbb{P}, \mathbb{H})\right)$, then

$$
\int_{-\infty}^{t} \mathcal{K}(t-s) \psi(s) d s=\int_{-\infty}^{t} \mathcal{K}(t-s) \psi_{1}(s) d s+\int_{-\infty}^{t} \mathcal{K}(t-s) \psi_{2}(s) d s:=\Theta_{1}(t)+\Theta_{2}(t)
$$

where

$$
\Theta_{1}(\mathrm{t})=\int_{-\infty}^{\mathrm{t}} \mathcal{K}(\mathrm{t}-\mathrm{s}) \psi_{1}(\mathrm{~s}) \mathrm{d} s, \quad \Theta_{2}(\mathrm{t})=\int_{-\infty}^{\mathrm{t}} \mathcal{K}(\mathrm{t}-\mathrm{s}) \psi_{2}(\mathrm{~s}) \mathrm{d} s
$$

It is easy to check that $\Theta_{1} \in \mathcal{U P C}\left(\mathbb{R}, \mathbb{L}^{2}(\mathbb{P}, \mathbb{H})\right)$. Since $\psi_{1} \in \mathcal{A} \mathcal{P}^{\mathfrak{p}}\left(\mathbb{R}, \mathbb{L}^{2}(\mathbb{P}, \mathbb{H})\right)$, for $\epsilon>0$, there exists a relatively dense set $R_{\epsilon}$ of $\mathbb{R}$ formed by $\epsilon$-periods of $\psi_{1}$. For $\tau \in R_{\epsilon}, t \in \mathbb{R},\left|t-t_{i}\right|>\epsilon, i \in \mathbb{Z}$, we have $\left\|\psi_{1}(t+\tau)-\psi_{1}(t)\right\|<\epsilon$.

Hence for $t \in \mathbb{R},\left|t-t_{i}\right|>\epsilon, i \in \mathbb{Z}$, we get

$$
\begin{aligned}
\mathbb{E}\left\|\Theta_{1}(t+\tau)-\Theta_{1}(t)\right\|^{2} & =\mathbb{E}\left\|\int_{-\infty}^{t+\tau} \mathcal{K}(t+\tau-s) \psi_{1}(s) d s-\int_{-\infty}^{t} \mathcal{K}(t-s) \psi_{1}(s) d s\right\|^{2} \\
& \leq \mathbb{E}\left\|\int_{-\infty}^{t} \mathcal{K}(t-s)\left[\psi_{1}(s+\tau)-\psi_{1}(s)\right] \mathrm{d} s\right\|^{2} \\
& \leq C_{\mathcal{K}}^{2} \int_{-\infty}^{t} e^{-2 b(t-s)} \mathbb{E}\left\|\psi_{1}(s+\tau)-\psi_{1}(s)\right\|^{2} \mathrm{~d} s<\frac{C_{\mathcal{K}}^{2}}{2 b} \epsilon
\end{aligned}
$$

which implies that $\Theta_{1} \in \mathcal{A P}^{\mathfrak{p}}\left(\mathbb{R}, \mathbb{L}^{2}(\mathbb{P}, \mathbb{H})\right)$. 
Next we show that $\Theta_{2} \in \mathcal{P} \mathcal{A} \mathcal{P}_{\rho}\left(\mathbb{R}, \mathbb{L}^{2}(\mathbb{P}, \mathbb{H})\right)$. In fact for $\gamma>0$, we have

$$
\begin{aligned}
\frac{1}{\mu(\gamma, \rho)} \int_{-\gamma}^{\gamma} \mathbb{E}\left\|\Theta_{2}(t)\right\|^{2} \rho(t) d t & =\frac{1}{\mu(\gamma, \rho)} \int_{-\gamma}^{\gamma} \mathbb{E}\left\|\int_{-\infty}^{t} \mathcal{K}(t-s) \psi_{2}(s) d s\right\|^{2} \rho(t) d t \\
& =\frac{1}{\mu(\gamma, \rho)} \int_{-\gamma}^{\gamma} \mathbb{E}\left\|\int_{0}^{\infty} \mathcal{K}(s) \psi_{2}(t-s) d s\right\|^{2} \rho(t) d t \\
& \leq \frac{C_{\mathcal{K}}^{2}}{\mu(\gamma, \rho)} \int_{-\gamma}^{\gamma} \int_{0}^{\infty} e^{-2 b(s)} \rho(t) \mathbb{E}\left\|\psi_{2}(t-s)\right\|^{2} d s d t \\
& \leq C_{\mathcal{K}}^{2} \int_{0}^{\infty} e^{-2 b(s)} \Lambda_{\gamma}(s) d s
\end{aligned}
$$

where

$$
\Lambda_{\gamma}(s)=\frac{1}{\mu(\gamma, \rho)} \int_{-\gamma}^{\gamma} \rho(t) \mathbb{E}\left\|\psi_{2}(t-s)\right\|^{2} d t
$$

Since $\psi_{2}(s) \in \mathcal{P} \mathcal{A} \mathcal{P}_{\rho}\left(\mathbb{R}, \mathbb{L}^{2}(\mathbb{P}, \mathbb{H})\right), \rho \in W_{\mathrm{T}}$, this implies that $\psi_{2}(\cdot-s) \in \mathcal{P} \mathcal{A} \mathcal{P} \rho\left(\mathbb{R}, \mathbb{L}^{2}(\mathbb{P}, \mathbb{H})\right)$ for each $s \in \mathbb{R}$ by Remark 3. Hence $\lim _{\gamma \rightarrow \infty} \Lambda_{\gamma}(s)=0$ for all $s \in \mathbb{R}$. Now, by Lebesgue dominated convergence theorem, we have $\Theta_{2} \in \mathcal{P} \mathcal{A} \mathcal{P} \rho\left(\mathbb{R}, \mathbb{L}^{2}(\mathbb{P}, \mathbb{H})\right)$.

Lemma 3.2. Assume that $\left(\mathrm{H}_{1}\right)-\left(\mathrm{H}_{2}\right)$ hold, if $\phi(\mathrm{t}) \in \mathcal{W} \mathcal{P} \mathcal{A} \mathcal{P}_{\rho}\left(\mathbb{R}, \mathbb{L}^{2}\left(\mathrm{P}, \mathrm{L}_{2}^{0}\right)\right)$, then

$$
\Lambda_{\phi}(t)=\int_{-\infty}^{t} A^{\sigma}(t-s)^{\alpha-1} \mathcal{J}(t-s) \phi(s) d w(s) \in \mathcal{W} \mathcal{P} \mathcal{A} \mathcal{P}_{\rho}\left(\mathbb{R}, \mathbb{L}^{2}(\mathbb{P}, \mathbb{H})\right)
$$

Proof. Since $\phi(t) \in \mathcal{W P} \mathcal{A} \mathcal{P}_{\rho}\left(\mathbb{R}, \mathbb{L}^{2}\left(\mathbb{P}, \mathbb{L}_{2}^{0}\right)\right)$ and $\|\phi\|_{\infty}:=\sup _{t \in \mathbb{R}}\left(\mathbb{E}\|\phi(t)\|^{2}\right)^{\frac{1}{2}}<\infty$. Now, using Ito's isometry property of stochastic integral and Lemma 2.1 , we get

$$
\begin{aligned}
\mathbb{E}\left\|\Lambda_{\phi}(t)\right\|^{2} & =\mathbb{E}\left\|\int_{-\infty}^{t} A^{\sigma}(t-s)^{\alpha-1} \mathcal{J}(t-s) \phi(s) \mathrm{d} w(s)\right\|^{2} \\
& \leq \alpha^{2} \mathbb{E}\left\|\int_{-\infty}^{t} \int_{0}^{\infty} \theta(t-s)^{\alpha-1} \mathcal{N}_{\alpha}(\theta) A^{\sigma} \mathcal{S}\left((t-s)^{\alpha} \theta\right) \phi(s) d \theta d w(s)\right\|^{2} \\
& \leq \alpha^{2}\left[\int_{-\infty}^{t} \int_{0}^{\infty} \mathbb{E}\left\|\theta(t-s)^{\alpha-1} \mathcal{N}_{\alpha}(\theta) A^{\sigma} \mathcal{S}\left((t-s)^{\alpha} \theta\right) \phi(s)\right\|^{2} \mathrm{~d} \theta \mathrm{d} s\right] \\
& \leq \alpha^{2} M_{\sigma}^{2} \int_{-\infty}^{t} \int_{0}^{\infty} \theta^{2(1-\sigma)} \mathcal{N}_{\alpha}^{2}(\theta)(t-s)^{2(\alpha-\alpha \sigma-1)} e^{2 \lambda \theta(t-s)^{\alpha}} \mathbb{E}\|\phi(s)\|^{2} \mathrm{~d} \theta \mathrm{d} s \\
& \leq \alpha^{2} M_{\sigma}^{2}\|\phi\|^{2} \int_{0}^{\infty} \mathcal{N}_{\alpha}^{2}(\theta) \int_{0}^{\infty} \theta^{2(1-\sigma)} \xi^{2(\alpha-\alpha \sigma-1)} e^{2 \lambda \theta \xi^{\alpha}} \mathrm{d} \xi \mathrm{d} \theta .
\end{aligned}
$$

Since $\mathcal{N}_{\alpha}^{2}(\theta) \in \mathrm{L}^{1}\left(\mathrm{R}^{+}\right)$, then by calculating we get (see [11])

$$
\alpha^{2} \int_{0}^{\infty} \mathcal{N}_{\alpha}^{2}(\theta) \int_{0}^{\infty} \theta^{2(1-\sigma)} \xi^{2(\alpha-\alpha \sigma-1)} e^{2 \lambda \theta \xi^{\alpha}} \mathrm{d} \xi \mathrm{d} \theta=\mathcal{N}_{0} \frac{\Gamma(1-2 \sigma)}{\lambda^{(2-2 \sigma)}}
$$

where $\mathcal{N}_{0}=\sup _{\theta \geq 0} \mathcal{N}_{\alpha}^{2}(\theta)$. Then

$$
\mathbb{E}\left\|\Lambda_{\phi}(t)\right\|^{2} \leq M_{\sigma}^{2}\|\phi\|^{2} \mathcal{N}_{0} \frac{\Gamma(1-2 \sigma)}{\lambda^{(2-2 \sigma)}},
$$


This implies that $\Lambda_{\phi}$ is well defined. Now let $\phi=\phi_{1}+\phi_{2}$, with $\phi_{1} \in \mathcal{A P}^{\mathrm{p}}\left(\mathbb{R}, \mathbb{L}^{2}(\mathbb{P}, \mathbb{H})\right)$ and $\phi_{2} \in \mathcal{P} \mathcal{A} \mathcal{P}_{\rho}\left(\mathbb{R}, \mathbb{L}^{2}(\mathbb{P}, \mathbb{H})\right)$, then

$$
\begin{aligned}
\Lambda_{\phi}(t) & =\int_{-\infty}^{t} A^{\sigma}(t-s)^{\alpha-1} \mathcal{J}(t-s) \phi_{1}(s) d w(s)+\int_{-\infty}^{t} A^{\sigma}(t-s)^{\alpha-1} \mathcal{J}(t-s) \phi_{2}(s) d w(s) \\
& :=\Lambda_{\phi_{1}}(t)+\Lambda_{\phi_{2}}(t) .
\end{aligned}
$$

It is easy to check that $\Lambda_{\phi_{1}} \in \mathcal{U P C}\left(\mathbb{R}, \mathbb{L}^{2}(\mathbb{P}, \mathbb{H})\right)$. Since $\phi_{1} \in \mathcal{A P}^{\mathfrak{p}}\left(\mathbb{R}, \mathbb{L}^{2}(\mathbb{P}, \mathbb{H})\right)$, for $\epsilon>0$, there exists a relatively dense set $R_{\epsilon}$ of $\mathbb{R}$ such that $\mathbb{E}\left\|\phi_{1}(t+\tau)-\phi_{1}(t)\right\|^{2}<\epsilon$ for $\tau \in R_{\epsilon}, t \in \mathbb{R},\left|t-t_{i}\right|>$ $\epsilon, i \in \mathbb{Z}$,

Note that $\widetilde{\mathcal{w}}(\mathrm{s}):=\mathfrak{w}(\mathrm{s}+\tau)-\mathcal{w}(\mathrm{s}), \mathrm{s} \in \mathbb{R}$, is also a Brownian motion with same distribution as $w(s)$. Now for $t \in \mathbb{R},\left|t-t_{i}\right|>\epsilon, i \in \mathbb{Z}$, using Lemma 2.1 and Ito's isometry property of stochastic integral, we have

$$
\begin{aligned}
& \mathbb{E}\left\|\Lambda_{\phi_{1}}(t+\tau)-\Lambda_{\phi_{1}}(t)\right\|^{2} \\
&= \mathbb{E}\left\|\int_{-\infty}^{t}(t-s)^{\alpha-1} A^{\sigma} \mathcal{J}_{\alpha}(t-s)\left[\phi_{1}(s+\tau)-\phi_{1}(s)\right] \mathrm{d} \widetilde{w}(s)\right\|^{2} \\
& \leq \alpha^{2} \mathbb{E}\left\|\int_{-\infty}^{t} \int_{0}^{\infty} \theta(t-s)^{\alpha-1} \mathcal{N}_{\alpha}(\theta) A^{\sigma} \mathcal{S}\left((t-s)^{\alpha} \theta\right)\left[\phi_{1}(s+\tau)-\phi_{1}(s)\right] \mathrm{d} \theta \mathrm{d} \widetilde{w}(s)\right\|^{2} \\
& \leq \alpha^{2} M_{\sigma}^{2} \int_{-\infty}^{t} \int_{0}^{\infty} \theta^{2(1-\sigma)} \mathcal{N}_{\alpha}^{2}(\theta)(t-s)^{2(\alpha-\sigma \alpha-1)} e^{-2 \lambda \theta(t-s)^{\alpha}} \mathbb{E}\left\|\left[\phi_{1}(s+\tau)-\phi_{1}(s)\right]\right\|^{2} \mathrm{~d} \theta \mathrm{d} s \\
&<\epsilon \alpha^{2} M_{\sigma}^{2} \int_{-\infty}^{t} \int_{0}^{\infty} \theta^{2(1-\sigma)} \mathcal{N}_{\alpha}^{2}(\theta)(t-s)^{2(\alpha-\sigma \alpha-1)} e^{-2 \lambda \theta(t-s)^{\alpha}} \mathrm{d} \theta \mathrm{d} s . \\
& \leq \epsilon \alpha^{2} M_{\sigma}^{2} \int_{0}^{\infty} \mathcal{N}_{\alpha}^{2}(\theta) \int_{0}^{\infty} \theta^{2(1-\sigma)} \xi^{2(\alpha-\sigma \alpha-1)} e^{-2 \lambda \theta \xi^{\alpha}} \mathrm{d} \xi \mathrm{d} \theta \\
& \leq M_{\sigma}^{2} \mathcal{N}_{0} \frac{\Gamma(1-2 \sigma)}{\lambda^{(2-2 \sigma)}} \epsilon
\end{aligned}
$$

that is $\Lambda_{\phi_{1}} \in \mathcal{A} \mathcal{P}^{\mathrm{p}}\left(\mathbb{R}, \mathbb{L}^{2}(\mathbb{P}, \mathbb{H})\right)$.

Next we show that $\Lambda_{\phi_{2}} \in \mathcal{P} \mathcal{A} \mathcal{P}_{\rho}\left(\mathbb{R}, \mathbb{L}^{2}(\mathbb{P}, \mathbb{H})\right)$. In fact for $\gamma>0$, we have

$$
\begin{aligned}
\frac{1}{\mu(\gamma, \rho)} \int_{-\gamma}^{\gamma} \mathbb{E}\left\|\Lambda_{\phi_{2}}(t)\right\|^{2} \rho(t) d t & =\frac{1}{\mu(\gamma, \rho)} \int_{-\gamma}^{\gamma} \mathbb{E}\left\|\int_{-\infty}^{t} A^{\sigma}(t-s)^{\alpha-1} \mathcal{J}(t-s) \phi_{2}(s) d w(s)\right\|^{2} \rho(t) d t \\
& \leq \frac{1}{\mu(\gamma, \rho)} \int_{-\gamma}^{\gamma} \int_{-\infty}^{t}\left\|A^{\sigma}(t-s)^{\alpha-1} \mathcal{J}(t-s)\right\|^{2} \mathbb{E}\left\|\phi_{2}(s)\right\|^{2} d s \rho(t) d t \\
& \leq \frac{1}{\mu(\gamma, \rho)} \int_{-\gamma}^{\gamma} \int_{0}^{\infty}\left\|\xi^{\alpha-1} A^{\sigma} \mathcal{J}(\xi)\right\|^{2} \mathbb{E}\left\|\phi_{2}(t-\xi)\right\|^{2} d \xi \rho(t) d t .
\end{aligned}
$$


Similar as previous calculation, we have

$$
\begin{array}{rl}
\int_{0}^{\infty}\left\|\xi^{\alpha-1} A^{\sigma} \mathcal{J}(\xi)\right\|^{2} & \mathbb{E}\left\|\phi_{2}(t-\xi)\right\|^{2} \mathrm{~d} \xi \\
\leq & \leq \alpha^{2} M_{\sigma}^{2} \int_{0}^{\infty} \mathcal{N}_{\alpha}^{2}(\theta) \int_{0}^{\infty} \theta^{2(1-\sigma)} \xi^{2(\alpha-\sigma \alpha-1)} e^{-2 \lambda \theta \xi^{\alpha}} \mathbb{E}\left\|\phi_{2}(t-\xi)\right\|^{2} \mathrm{~d} \xi \mathrm{d} \theta
\end{array}
$$

Now, we have

$$
\frac{1}{\mu(\gamma, \rho)} \int_{-\gamma}^{\gamma} \mathbb{E}\left\|\Lambda_{2} y(t)\right\|^{2} \rho(t) d t \leq \alpha^{2} M_{\sigma}^{2} \int_{0}^{\infty} \mathcal{N}_{\alpha}^{2}(\theta) \int_{0}^{\infty} \theta^{2(1-\sigma)} \xi^{2(\alpha-\sigma \alpha-1)} e^{-2 \lambda \theta \xi^{\alpha}} \mathbb{T}_{\gamma}(t) d \xi d \theta .
$$

where

$$
\mathbb{T}_{\gamma}(\mathrm{t})=\frac{1}{\mu(\gamma, \rho)} \int_{-\gamma}^{\gamma} \mathbb{E}\left\|\phi_{2}(\mathrm{t}-\xi)\right\|^{2} \rho(\mathrm{t}) \mathrm{dt} .
$$

Since $\phi_{2}(s) \in \mathcal{P} \mathcal{A} \mathcal{P}_{\rho}\left(\mathbb{R}, \mathbb{L}^{2}(\mathbb{P}, \mathbb{H})\right), \rho \in W_{\mathrm{T}}$ and translation invariant, this implies that $\phi_{2}(\cdot-s) \in$ $\mathcal{P} \mathcal{A} \mathcal{P}_{\rho}\left(\mathbb{R}, \mathbb{L}^{2}(\mathbb{P}, \mathbb{H})\right)$ for each $s \in \mathbb{R}$ by Remark 3. Hence ${ }_{\gamma \rightarrow \infty} \mathbb{T}_{\gamma}(t)=0$ for all $s \in \mathbb{R}$. Now, by Lebesgue dominated convergence theorem, we have $\Lambda_{\phi_{2}} \in \mathcal{P} \mathcal{A} \mathcal{P}_{\rho}\left(\mathbb{R}, \mathbb{L}^{2}(\mathbb{P}, \mathbb{H})\right)$.

Theorem 3.1. Assume the conditions $\left(\mathrm{H}_{1}\right)-\left(\mathrm{H}_{7}\right)$ are satisfy, if

$$
\Delta:=3 \mathrm{M}_{\sigma}^{2}\left[4 \mathrm{~L}_{\mathcal{G}} \mathrm{N}_{0}^{2}\left(\frac{1}{M_{0}^{\sigma}}+\frac{1}{e^{\lambda}-1}\right)^{2}+\mathrm{L}_{\mathrm{G}} \frac{\Gamma^{2}(1-\sigma)}{\lambda^{2(1-\sigma)}}+\mathrm{L}_{\mathrm{F}} \mathcal{N}_{0}\left(1+\frac{\mathrm{L}_{g} \mathrm{C}_{\mathcal{K}}^{2}}{2 \mathrm{~b}}\right) \frac{\Gamma(1-2 \sigma)}{\lambda^{(2-2 \sigma)}}\right]<1,
$$

then the system $(1.1)-(1.3)$ admits a unique mild solution in $\mathcal{W P} \mathcal{A} \mathcal{P} \rho\left(\mathbb{R}, \mathbb{L}^{2}(\mathbb{P}, \mathbb{H})\right)$.

Proof. Let $\mathcal{M}:=\left\{y \in \mathcal{W} \mathcal{P} \mathcal{A} \mathcal{P}_{\rho}\left(\mathbb{R}, \mathbb{L}^{2}(\mathbb{P}, \mathbb{H})\right)\right.$ with discontinuity of first type at $t_{i}, i \in \mathbb{Z}$ satisfying $\left.\mathbb{E}\|y\|^{2} \leq r_{0}, r_{0}>0\right\}$. Obviously, $\mathcal{M}$ is a closet subspace of $\mathcal{W P} \mathcal{A} \mathcal{P}_{\rho}\left(\mathbb{R}, \mathbb{L}^{2}(\mathbb{P}, \mathbb{H})\right)$. Define an operator $\mathcal{Q}$ in $\mathcal{M}$ by

$$
\begin{aligned}
(\mathcal{Q} y)(t)= & \sum_{t_{i}<t} A^{\sigma} \mathcal{I}\left(t-t_{i}\right) \mathcal{G}_{i}\left(A^{-\sigma} y\left(t_{i}\right)\right)+\int_{-\infty}^{t}(t-s)^{\alpha-1} A^{\sigma} \mathcal{J}(t-s) G\left(s, A^{-\sigma} y(s) d s\right. \\
& +\int_{-\infty}^{t}(t-s)^{\alpha-1} A^{\sigma} \mathcal{J}(t-s) F\left(s, A^{-\sigma} y(s), \Theta\left(A^{-\sigma} y(s)\right)\right) d w(s) .
\end{aligned}
$$

where $\Theta\left(A^{-\sigma} y(s)\right)=\int_{-\infty}^{s} \mathcal{K}(s-\xi) g\left(\xi, A^{-\sigma} y(\xi)\right) d \xi$.

In fact if $y \in \mathcal{W P} \mathcal{A} \mathcal{P}\left(\mathbb{R}, \mathbb{L}^{2}\left(\mathbb{P}, \mathbb{H}_{\sigma}\right)\right)$, we have $\Theta\left(A^{-\sigma} y\right) \in \mathcal{W P} \mathcal{A P} \rho\left(\mathbb{R}, \mathbb{L}^{2}(\mathbb{P}, \mathbb{H})\right)$ by Lemma 3.1, and $\mathrm{F}\left(\cdot, \mathrm{A}^{-\sigma} \mathrm{y}(\cdot), \Theta\left(\mathrm{A}^{-\sigma} \mathrm{y}(\cdot)\right)\right) \in \mathcal{W} \mathcal{P} \mathcal{A} \mathcal{P}\left(\mathbb{R}, \mathbb{L}^{2}\left(\mathbb{P}, \mathbb{L}_{2}^{0}\right)\right)$ by Theorem 2.2. Further by Theorem 3.2 we get $\int_{-\infty}^{t}(t-s)^{\alpha-1} A^{\sigma} \mathcal{J}(t-s) F\left(s, A^{-\sigma} y(s), \Theta\left(A^{-\sigma} y(s)\right)\right) d w(s) \in \mathcal{W} \mathcal{P} \mathcal{A} \mathcal{P}_{\rho}\left(\mathbb{R}, \mathbb{L}^{2}(\mathbb{P}, \mathbb{H})\right)$. Similarly by Theorem 2.2 and Theorem 3.2 we come to the conclusion that $\int_{-\infty}^{t}(t-s)^{\alpha-1} A^{\sigma} \mathcal{J}(t-$ s) $G\left(s, A^{-\sigma} y(s) d s \in \mathcal{W P} \mathcal{A} \mathcal{P}_{\rho}\left(\mathbb{R}, \mathbb{L}^{2}(\mathbb{P}, \mathbb{H})\right)\right.$.

Further, we show that

$$
\sum_{t_{i}<t} A^{\sigma} \mathcal{I}\left(t-t_{i}\right) \mathcal{G}_{i}\left(A^{-\sigma} y\left(t_{i}\right)\right) \in \mathcal{W P} \mathcal{A} \mathcal{P}_{\rho}\left(\mathbb{R}, \mathbb{L}^{2}(\mathbb{P}, \mathbb{H})\right)
$$


By Theorem 2.2, $\mathcal{G}_{\mathfrak{i}}\left(A^{-\sigma} \mathrm{y}\left(\mathrm{t}_{\mathrm{i}}\right)\right) \in \mathcal{W} \mathcal{P} \mathcal{A} \mathcal{P}_{\rho}\left(\mathbb{Z}, \mathbb{L}^{2}(\mathbb{P}, \mathbb{H})\right)$. Now, let $\mathcal{G}_{i}\left(A^{-\sigma} \mathrm{y}\left(\mathrm{t}_{i}\right)\right)=\phi_{i}+\psi_{i}$, where $\phi_{i} \in \mathcal{A P}\left(\mathbb{Z}, \mathbb{L}^{2}(\mathbb{P}, \mathbb{H})\right)$ and $\psi_{i} \in \mathcal{P} \mathcal{A P} \mathcal{P}_{\rho}\left(\mathbb{Z}, \mathbb{L}^{2}(\mathbb{P}, \mathbb{H})\right)$ for all $i \in \mathbb{Z}$, so

$$
\begin{aligned}
\sum_{t_{i}<t} A^{\sigma} \mathcal{I}\left(t-t_{i}\right) \mathcal{G}_{i}\left(A^{-\sigma} y\left(t_{i}\right)\right) & =\sum_{t_{i}<t} A^{\sigma} \mathcal{I}\left(t-t_{i}\right) \phi_{i}+\sum_{t_{i}<t} A^{\sigma} \mathcal{I}\left(t-t_{i}\right) \psi_{i} \\
& :=\Upsilon_{1}(t)+\Upsilon_{2}(t) .
\end{aligned}
$$

Since $\left\{t_{i}^{j}: i, j \in \mathbb{Z}\right\}$ is equipotentially almost periodic, then by Lemma 2.2, for $\epsilon>0$ there exists relative dense set $R_{\epsilon}$ of real numbers and $Z_{\epsilon}$ of integers, such that for $t_{i}<t<t_{i+1}, \tau \in R_{\epsilon}$, $p \in Z_{\epsilon},\left|t-t_{i}\right|>\epsilon,\left|t-t_{i+1}\right|>\epsilon, i \in \mathbb{Z}$, we have

$$
\begin{array}{r}
t+\tau>t_{i}+\epsilon+\tau>t_{i+p}, \\
t_{i+p+1}>t_{i+1}+\tau-\epsilon>t+\tau,
\end{array}
$$

that is, $t_{i+p}<t+\tau<t_{i+p+1}$, then using Cauchy-Schwarz inequality we have

$$
\begin{aligned}
\mathbb{E}\left\|\Upsilon_{1}(t+\tau)-\Upsilon_{1}(t)\right\|^{2} & \leq \mathbb{E}\left\|\sum_{t_{i}<t+\tau} A^{\sigma} \mathcal{I}\left(t+\tau-t_{i}\right) \phi_{i}-\sum_{t_{i}<t} A^{\sigma} \mathcal{I}\left(t-t_{i}\right) \phi_{i}\right\|^{2} \\
& \leq \mathbb{E}\left[\sum_{t_{i}<t}\left\|A^{\sigma} \mathcal{I}\left(t-t_{i}\right)\left[\phi_{i+p}-\phi_{i}\right]\right\|\right]^{2} \\
& \leq \sum_{t_{i}<t} \mathbb{E}\left[\int_{0}^{\infty} \mathcal{N}_{\alpha}(\theta) A^{\sigma} \mathcal{S}\left(\left(t-t_{i}\right)^{\alpha} \theta\right)\left\|\phi_{i+p}-\phi_{i}\right\| d \theta\right]^{2} \\
& \leq \sum_{t_{i}<t} \mathbb{E}\left[\int_{0}^{\infty} \mathcal{N}_{\alpha}(\theta) M_{\sigma}\left(\left(t-t_{i}\right)^{\alpha} \theta\right)^{-\sigma} e^{-\lambda\left(t-t_{i}\right)^{\alpha} \theta}\left\|\phi_{i+p}-\phi_{i}\right\| d \theta\right]^{2} \\
& \leq M_{\sigma}^{2} \sum_{t_{i}<t} \mathbb{E}\left[\int_{0}^{\infty} \mathcal{N}_{\alpha}(\theta)\left(\left(t-t_{i}\right)^{\alpha} \theta\right)^{-\sigma} e^{-\lambda\left(t-t_{i}\right)^{\alpha} \theta}\left\|\phi_{k+p}-\phi_{i}\right\| d \theta\right]^{2} \\
& <\in M_{\sigma}^{2} R(\theta),
\end{aligned}
$$

where

$$
\begin{aligned}
R(\theta)= & \left(\int _ { 0 } ^ { \infty } \mathcal { N } _ { \alpha } ( \theta ) \left[\sum_{0<t-t_{i} \leq 1}\left(\left(t-t_{i}\right)^{\alpha} \theta\right)^{-\sigma} e^{-\lambda\left(t-t_{i}\right)^{\alpha} \theta}\right.\right. \\
& \left.\left.+\sum_{l=1}^{\infty} \sum_{l<t-t_{i} \leq l+1}\left(\left(t-t_{i}\right)^{\alpha} \theta\right)^{-\sigma} e^{-\lambda\left(t-t_{i}\right)^{\alpha} \theta}\right] d \theta\right)^{2} .
\end{aligned}
$$

By Lemma 2.4 and $\left(\mathrm{H}_{1}\right)$, we have

$$
R(\theta) \leq\left(\int_{0}^{\infty} \mathcal{N}_{\alpha}(\theta)\left[\frac{2 N_{0}}{M_{0}^{\sigma}}+\frac{2 N_{0}}{e^{\lambda}-1}\right] d \theta\right)^{2}=4 N_{0}^{2}\left(\frac{1}{M_{0}^{\sigma}}+\frac{1}{e^{\lambda}-1}\right)^{2},
$$

where $M_{0}=\min \left\{\left(t-t_{i}\right)^{\alpha} \theta, 0<t-t_{i} \leq 1\right\}$. Then

$$
\mathbb{E}\left\|\Upsilon_{1}(t+\tau)-\Upsilon_{1}(t)\right\|^{2}<4 \in M_{\sigma}^{2} N_{0}^{2}\left(\frac{1}{M_{0}^{\sigma}}+\frac{1}{e^{\lambda}-1}\right)^{2}
$$


Hence $\Upsilon_{1}(\mathrm{t}) \in \mathcal{A P}^{\mathrm{p}}\left(\mathbb{R}, \mathrm{L}^{2}(\mathbb{P}, \mathbb{H})\right)$.

Next, we show that $\Upsilon_{2}(t) \in \mathcal{P} \mathcal{A} \mathcal{P}_{\rho}\left(\mathbb{R}, \mathbb{L}^{2}(\mathbb{P}, \mathbb{H})\right)$. Define

$$
x(t)=A^{\sigma} \mathcal{I}\left(t-t_{i}\right) \psi_{i}, \quad t \in\left(t_{i}, t_{i+1}\right], i \in \mathbb{Z},
$$

then

$$
\begin{aligned}
\lim _{t \rightarrow \infty} \mathbb{E}\|x(t)\|^{2} & =\lim _{t \rightarrow \infty} \mathbb{E}\left\|A^{\sigma} \mathcal{I}\left(t-t_{i}\right) \psi_{i}\right\|^{2} \\
& \leq \lim _{t \rightarrow \infty} \mathbb{E}\left[\int_{0}^{\infty} \mathcal{N}_{\alpha}(\theta) A^{\sigma} \mathcal{S}\left(\left(t-t_{i}\right)^{\alpha} \theta\right)\left\|\psi_{i}\right\| d \theta\right]^{2} \\
& \leq \lim _{t \rightarrow \infty} M_{\sigma}^{2} \int_{0}^{\infty} \mathcal{N}_{\alpha}^{2}(\theta)\left(\left(t-t_{i}\right)^{\alpha} \theta\right)^{-2 \sigma} e^{-2 \lambda\left(t-t_{i}\right)^{\alpha}} \theta \mathbb{E}\left\|\psi_{i}\right\|^{2} d \theta \\
& \leq \lim _{t \rightarrow \infty} M_{\sigma}^{2} \mathbb{E}\left\|\psi_{i}\right\|^{2}\left(t-t_{i}\right)^{-2 \alpha \sigma} \int_{0}^{\infty} \mathcal{N}_{\alpha}^{2}(\theta) \theta^{-2 \sigma} e^{-2 \lambda\left(t-t_{i}\right)^{\alpha} \theta} d \theta=0
\end{aligned}
$$

then $x \in \mathcal{P} \mathcal{A} \mathcal{P}_{\rho}\left(\mathbb{R}, \mathbb{L}^{2}(\mathbb{P}, \mathbb{H})\right)$. Define $x_{i}: \mathbb{R} \rightarrow \mathbb{L}^{2}(\mathbb{P}, \mathbb{H})$ by

$$
x_{n}(t)=A^{\sigma} \mathcal{I}\left(t-t_{i-n}\right) \psi_{i-n}, \quad t \in\left(t_{i}, t_{i+1}\right], n \in \mathbb{Z}^{+},
$$

so, $x_{n} \in \mathcal{P} \mathcal{A} \mathcal{P}_{\rho}\left(\mathbb{R}, \mathbb{L}^{2}(\mathbb{P}, \mathbb{H})\right)$. Moreover

$$
\begin{aligned}
\mathbb{E}\left\|x_{n}(t)\right\|^{2} & =\mathbb{E}\left\|A^{\sigma} \mathcal{I}\left(t-t_{i-n}\right) \psi_{i-n}\right\|^{2} \\
& \leq \mathbb{E}\left[\int_{0}^{\infty} \mathcal{N}_{\alpha}(\theta) A^{\sigma} \mathcal{S}\left(\left(t-t_{i-n}\right)^{\alpha} \theta\right)\left\|\psi_{i-n}\right\| d \theta\right]^{2} \\
& \leq M_{\sigma}^{2} \int_{0}^{\infty} \mathcal{N}_{\alpha}^{2}(\theta)\left(\left(t-t_{i-n}\right)^{\alpha} \theta\right)^{-2 \sigma} e^{-2 \lambda\left(t-t_{i-n}\right)^{\alpha} \theta} \mathbb{E}\left\|\psi_{i-n}\right\|^{2} d \theta \\
& \leq M_{\sigma}^{2} C_{0} \int_{0}^{\infty} \mathcal{N}_{\alpha}^{2}(\theta)\left(\left(t-t_{i}+n k\right)^{\alpha} \theta\right)^{-2 \sigma} e^{-2 \lambda\left(t-t_{i}+n k\right)^{\alpha} \theta} d \theta \\
& \leq M_{\sigma}^{2} C_{0} \int_{0}^{\infty} \mathcal{N}_{\alpha}^{2}(\theta)\left(t-t_{i}+n k\right)^{-2 \alpha \sigma} \theta^{-2 \sigma} e^{-2 \lambda\left(t-t_{i}+n k\right)^{\alpha} \theta} d \theta \\
& \leq \frac{M_{\sigma}^{2} C_{0}}{\left(t-t_{i}+n k\right)^{2 \alpha \sigma}} \int_{0}^{\infty} \mathcal{N}_{\alpha}^{2}(\theta) \theta^{-2 \sigma} e^{-2 \lambda\left(t-t_{i}+n k\right)^{\alpha} \theta} d \theta,
\end{aligned}
$$

where $\sup _{i \in \mathbb{Z}} \mathbb{E}\left\|\psi_{i}\right\|^{2}=\mathrm{C}_{0}$. Therefore by Dirchlet Test the series $\sum_{n=1}^{\infty} x_{n}$ converges uniformly on $\mathbb{R}$. By Lemma 2.3, we have

$$
\Upsilon_{2}(t)=\sum_{t_{i}<t} A^{\sigma} \mathcal{I}\left(t-t_{i}\right) \psi_{i}=\sum_{n=0}^{\infty} x_{n} \in \mathcal{W} \mathcal{P} \mathcal{A} \mathcal{P}_{\rho}\left(\mathbb{R}, \mathbb{L}^{2}(\mathbb{P}, \mathbb{H})\right)
$$

So, $(\mathcal{Q} y) \in \mathcal{W} \mathcal{P} \mathcal{A} \mathcal{P}_{\rho}\left(\mathbb{R}, \mathbb{L}^{2}(\mathbb{P}, \mathbb{H})\right)$. 
Moreover

$$
\begin{aligned}
& \mathbb{E}\|\mathcal{Q} y(t)\|_{\sigma}^{2} \\
& \leq 3 \mathbb{E}\left\|\sum_{t_{i}<t} A^{\sigma} \mathcal{I}\left(t-t_{i}\right) \mathcal{G}_{i}\left(A^{-\sigma} y\left(t_{i}\right)\right)\right\|^{2}+3 \mathbb{E}\left\|\int_{-\infty}^{t}(t-s)^{\alpha-1} A^{\sigma} \mathcal{J}(t-s) G\left(s, A^{-\sigma} y(s)\right) d s\right\|^{2} \\
& +3 \mathbb{E}\left\|\int_{-\infty}^{t}(t-s)^{\alpha-1} A^{\sigma} \mathcal{J}(t-s) F\left(s, A^{-\sigma} y(s), \Theta\left(A^{-\sigma} y(s)\right)\right) d w(s)\right\|^{2} \\
& \leq 3 M_{\sigma}^{2} \sum_{t_{i}<t} \mathbb{E}\left\|\int_{0}^{\infty} \mathcal{N}_{\alpha}(\theta)\left(\left(t-t_{i}\right)^{\alpha} \theta\right)^{-\sigma} e^{-\lambda\left(t-t_{i}\right)^{\alpha} \theta} \mathcal{G}_{i}\left(A^{-\sigma} y\left(t_{i}\right)\right) d \theta\right\|^{2} \\
& +3 \alpha^{2} M_{\sigma}^{2} \mathbb{E}\left[\int_{-\infty}^{t} \int_{0}^{\infty} \theta^{(1-\sigma)} \mathcal{N}_{\alpha}(\theta)(t-s)^{(\alpha-\sigma \alpha-1)} e^{-\lambda \theta(t-s)^{\alpha}}\left\|G\left(s, A^{-\sigma} y(s)\right)\right\| d \theta d s\right]^{2} \\
& +3 \alpha^{2} M_{\sigma}^{2} \int_{-\infty}^{t} \int_{0}^{\infty} \theta^{2(1-\sigma)} \mathcal{N}_{\alpha}^{2}(\theta)(t-s)^{2(\alpha-\sigma \alpha-1)} e^{-2 \lambda \theta(t-s)^{\alpha}} \mathbb{E}\left\|\mathrm{F}\left(s, A^{-\sigma} y(s), \Theta\left(A^{-\sigma} y(s)\right)\right)\right\|^{2} \mathrm{~d} \theta \mathrm{d} s \\
& \leq 3 M_{\sigma}^{2} \sum_{t_{i}<t} \mathbb{E}\left[\int_{0}^{\infty} \mathcal{N}_{\alpha}(\theta)\left(\left(t-t_{i}\right)^{\alpha} \theta\right)^{-\sigma} e^{-\lambda\left(t-t_{i}\right)^{\alpha} \theta}\left\|\mathcal{G}_{i}\left(A^{-\sigma} y\left(t_{i}\right)\right)\right\| d \theta\right]^{2} \\
& +3 \alpha^{2} M_{\sigma}^{2} \int_{0}^{\infty} \mathcal{N}_{\alpha}(\theta) \int_{-\infty}^{t} \theta^{(1-\sigma)}(t-s)^{(\alpha-\sigma \alpha-1)} e^{-\lambda \theta(t-s)^{\alpha}} d s d \theta \\
& \times \int_{0}^{\infty} \mathcal{N}_{\alpha}(\theta) \int_{-\infty}^{t} \theta^{(1-\sigma)}(\mathrm{t}-\mathrm{s})^{(\alpha-\sigma \alpha-1)} e^{-\lambda \theta(\mathrm{t}-\mathrm{s})^{\alpha}} \mathbb{E}\left\|\mathrm{G}\left(\mathrm{s}, \mathrm{A}^{-\sigma} \mathrm{y}(\mathrm{s})\right)\right\|^{2} \mathrm{~d} \mathrm{~d} \mathrm{~d} \theta \\
& +3 \alpha^{2} M_{\sigma}^{2} \int_{-\infty}^{t} \int_{0}^{\infty} \theta^{2(1-\sigma)} \mathcal{N}_{\alpha}^{2}(\theta)(t-s)^{2(\alpha-\sigma \alpha-1)} e^{-2 \lambda \theta(t-s)^{\alpha}} \mathbb{E}\left\|F\left(s, A^{-\sigma} y(s), \Theta\left(A^{-\sigma} y(s)\right)\right)\right\|^{2} d \theta d s
\end{aligned}
$$

For $\xi=t-s$, we have

$$
\begin{aligned}
\mathbb{E}\|\mathcal{Q} y(t)\|_{\sigma}^{2} \leq & 3 M_{\sigma}^{2} C_{\mathcal{G}_{i}}^{2}\left[\int_{0}^{\infty} \mathcal{N}_{\alpha}(\theta) \sum_{t_{i}<t}\left(\left(t-t_{i}\right)^{\alpha} \theta\right)^{-\sigma} e^{-\lambda\left(t-t_{i}\right)^{\alpha} \theta} d \theta\right]^{2} \\
& +3 M_{\sigma}^{2} C_{G}^{2}\left[\alpha \int_{0}^{\infty} \mathcal{N}_{\alpha}(\theta) \int_{0}^{\infty} \theta^{(1-\sigma)} \xi^{(\alpha-\sigma \alpha-1)} e^{-\lambda \theta \xi^{\alpha}} d \xi d \theta\right]^{2} \\
& +3 \alpha^{2} M_{\sigma}^{2} C_{F}^{2} \int_{0}^{\infty} \mathcal{N}_{\alpha}^{2}(\theta) \int_{0}^{\infty} \theta^{2(1-\sigma)} \xi^{2(\alpha-\sigma \alpha-1)} e^{-2 \lambda \theta \xi^{\alpha}} \xi d \theta .
\end{aligned}
$$

By standard calculation, we have

$$
\begin{aligned}
{\left[\alpha \int_{0}^{\infty} \mathcal{N}_{\alpha}(\theta) \int_{0}^{\infty} \theta^{(1-\sigma)}\right.} & \left.\xi^{(\alpha-\sigma \alpha-1)} e^{-\lambda \theta \xi^{\alpha}} \mathrm{d} \xi \mathrm{d} \theta\right]^{2} \\
& =\left[\frac{1}{\lambda^{1-\sigma}} \int_{0}^{\infty} \mathcal{N}_{\alpha}(\theta) \int_{0}^{\infty}\left(\lambda \theta \xi^{\alpha}\right)^{-\sigma} e^{-\lambda \theta \xi^{\alpha}} \mathrm{d}\left(\lambda \theta \xi^{\alpha}\right) \mathrm{d} \theta\right]^{2} \\
& =\frac{\Gamma^{2}(1-\sigma)}{\lambda^{2(1-\sigma)}}
\end{aligned}
$$


Recalling the results (3.3), (3.8) and (3.10) in (3.9), we have

$$
\mathbb{E}\|\mathcal{Q} y(t)\|_{\sigma}^{2} \leq 3 M_{\sigma}^{2}\left[4 C_{\mathcal{G}_{i}}^{2} N_{0}^{2}\left(\frac{1}{M_{0}^{\sigma}}+\frac{1}{e^{\lambda}-1}\right)^{2}+C_{G}^{2} \frac{\Gamma^{2}(1-\sigma)}{\lambda^{2(1-\sigma)}}+C_{F}^{2} \mathcal{N}_{0} \frac{\Gamma(1-2 \sigma)}{\lambda^{(2-2 \sigma)}}\right] \leq r_{0},
$$

this implies that $\mathcal{Q} y \in \mathcal{M}$. Thus $\mathcal{Q}$ is well defined.

Now we show that $\mathcal{Q}$ is contraction. For $y_{1}, y_{2} \in \mathcal{M}$, we have

$$
\begin{aligned}
& \mathbb{E}\left\|\mathcal{Q}_{1}(\mathrm{t})-\mathcal{Q} y_{2}(t)\right\|^{2} \\
& \leq 3 \mathbb{E}\left\|\sum_{t_{i}<t} A^{\sigma} \mathcal{I}\left(t-t_{i}\right)\left[\mathcal{G}_{i}\left(y_{1}\left(t_{i}\right)\right)-\mathcal{G}_{i}\left(y_{2}\left(t_{i}\right)\right)\right]\right\|^{2} \\
& +3 \mathbb{E}\left\|\int_{-\infty}^{t}(t-s)^{\alpha-1} A^{\sigma} \mathcal{J}(t-s)\left[G\left(s, A^{-\sigma} y_{1}(s)\right)-G\left(s, A^{-\sigma} y_{2}(s)\right)\right] d s\right\|^{2} \\
& +3 \mathbb{E} \| \int_{-\infty}^{t}(t-s)^{\alpha-1} A^{\sigma} \mathcal{J}(t-s)\left[F\left(s, A^{-\sigma} y_{1}(s), \Theta\left(A^{-\sigma} y_{1}(s)\right)\right)\right. \\
& \left.\quad-F\left(s, A^{-\sigma} y_{2}(s), \Theta\left(A^{-\sigma} y_{2}(s)\right)\right)\right] d w(s) \|^{2} \\
& \leq 3 M_{\sigma}^{2} \sum_{t_{i}<t} \mathbb{E}\left[\int_{0}^{\infty} \mathcal{N}_{\alpha}(\theta)\left(\left(t-t_{i}\right)^{\alpha} \theta\right)^{-\sigma} e^{-\lambda\left(t-t_{i}\right)^{\alpha} \theta}\left\|\mathcal{G}_{i}\left(y_{1}\left(t_{i}\right)\right)-\mathcal{G}_{i}\left(y_{2}\left(t_{i}\right)\right)\right\| d \theta\right]^{2} \\
& +3 \alpha^{2} M_{\sigma}^{2} \int_{0}^{\infty} \mathcal{N}_{\alpha}(\theta) \int_{-\infty}^{t} \theta^{(1-\sigma)}(t-s)^{(\alpha-\sigma \alpha-1)} e^{-\lambda \theta(t-s)^{\alpha}} d s d \theta \\
& \quad \times \int_{0}^{\infty} \mathcal{N}_{\alpha}(\theta) \int_{-\infty}^{t} \theta^{(1-\sigma)}(t-s)^{(\alpha-\sigma \alpha-1)} e^{-\lambda \theta(t-s)^{\alpha}} \mathbb{E}\left\|G\left(s, A^{-\sigma} y_{1}(s)\right)-G\left(s, A^{-\sigma} y_{2}(s)\right)\right\|^{2} d s d \theta \\
& +3 \alpha^{2} M_{\sigma}^{2} \int_{-\infty}^{t} \int_{0}^{\infty} \theta^{2(1-\sigma)} \mathcal{N}_{\alpha}^{2}(\theta)(t-s)^{2(\alpha-\sigma \alpha-1)} e^{-2 \lambda \theta(t-s)^{\alpha}} \mathbb{E} \| F\left(s, A^{-\sigma} y_{1}(s), \Theta\left(A^{-\sigma} y_{1}(s)\right)\right) \\
& \quad-F\left(s, A^{-\sigma} y_{2}(s), \Theta\left(A^{-\sigma} y_{2}(s)\right)\right) \|^{2} d \theta d s \\
& \leq 3 M_{\sigma}^{2}\left[L_{\mathcal{G}}\left(\int_{0}^{\infty} \mathcal{N}_{\alpha}(\theta) \sum_{t_{i}<t}\left(\left(t-t_{i}\right)^{\alpha} \theta\right)^{-\sigma} e^{-\lambda\left(t-t_{i}\right)^{\alpha} \theta} d \theta\right)^{2}\right. \\
& +L_{G}\left(\alpha \int_{0}^{\infty} \mathcal{N}_{\alpha}(\theta) \int_{0}^{\infty} \theta^{(1-\sigma)} \xi^{(\alpha-\sigma \alpha-1)} e^{-\lambda \theta \xi^{\alpha}} d \xi d \theta\right)^{2} \\
& \left.+L_{F}\left(1+\frac{L_{g} C_{\mathcal{K}}^{2}}{2 b}\right) \int_{0}^{\infty} \mathcal{N}_{\alpha}^{2}(\theta) \int_{0}^{\infty} \alpha^{2} \theta^{2(1-\sigma)} \xi^{2(\alpha-\sigma \alpha-1)} e^{-2 \lambda \theta \xi^{\alpha}} \mathrm{d} \theta d \xi\right] \mathbb{E}\left\|y_{1}(t)-y_{2}(t)\right\|^{2} . \\
&
\end{aligned}
$$

Recalling the results (3.3), (3.8) and (3.10), we have

$$
\begin{aligned}
\mathbb{E}\left\|\mathcal{Q} y_{1}(t)-\mathcal{Q} y_{2}(t)\right\|^{2} \leq & 3 M_{\sigma}^{2}\left[4 L_{\mathcal{G}} \mathrm{N}_{0}^{2}\left(\frac{1}{M_{0}^{\sigma}}+\frac{1}{e^{\lambda}-1}\right)^{2}+\mathrm{L}_{G} \frac{\Gamma^{2}(1-\sigma)}{\lambda^{2(1-\sigma)}}\right. \\
& \left.+\mathrm{L}_{\mathrm{F}} \mathcal{N}_{0}\left(1+\frac{\mathrm{L}_{\mathrm{g}} \mathrm{C}_{\mathcal{K}}^{2}}{2 \mathrm{~b}}\right) \frac{\Gamma(1-2 \sigma)}{\lambda^{(2-2 \sigma)}}\right] \sup _{\mathrm{t} \in \mathbb{R}} \mathbb{E}\left\|\mathrm{y}_{1}(\mathrm{t})-\mathrm{y}_{2}(\mathrm{t})\right\|^{2} .
\end{aligned}
$$


This implies that $\left\|\mathcal{Q} y_{1}-\mathcal{Q} y_{2}\right\|_{\infty} \leq \sqrt{\Delta}\left\|y_{1}-y_{2}\right\|_{\infty}$, where

$$
\Delta=3 \mathrm{M}_{\sigma}^{2}\left[4 \mathrm{~L}_{\mathcal{G}} \mathrm{N}_{0}^{2}\left(\frac{1}{M_{0}^{\sigma}}+\frac{1}{e^{\lambda}-1}\right)^{2}+\mathrm{L}_{G} \frac{\Gamma^{2}(1-\sigma)}{\lambda^{2(1-\sigma)}}+\mathrm{L}_{\mathrm{F}} \mathcal{N}_{\mathrm{O}}\left(1+\frac{\mathrm{L}_{g} \mathrm{C}_{\mathcal{K}}^{2}}{2 \mathrm{~b}}\right) \frac{\Gamma(1-2 \sigma)}{\lambda^{(2-2 \sigma)}}\right]<1 .
$$

Thus, $\mathcal{Q}$ is a contraction. Hence by Banach contraction principle the system (1.1) - (1.3), admits a unique mild solution $\widetilde{y} \in \mathcal{M}$ such that $\mathcal{Q} \widetilde{y}=\widetilde{y}$. Moreover, since $A^{\sigma}$ is closed, then we have

$$
\begin{aligned}
A^{-\sigma} \widetilde{y}(t)= & \sum_{t_{i}<t} \mathcal{I}\left(t-t_{i}\right) \mathcal{G}_{i}\left(A^{-\sigma} \widetilde{y}\left(t_{i}\right)\right)+\int_{-\infty}^{t}(t-s)^{\alpha-1} \mathcal{J}(t-s) G\left(s, A^{-\sigma} \widetilde{y}(s) d s\right. \\
& +\int_{-\infty}^{t}(t-s)^{\alpha-1} \mathcal{J}(t-s) F\left(s, A^{-\sigma} \widetilde{y}(s), \Theta\left(A^{-\sigma} \widetilde{y}(s)\right)\right) d w(s) .
\end{aligned}
$$

such that $\mathbb{E}\left\|A^{-\sigma} \widetilde{\mathfrak{y}}(t)\right\|^{2}<r_{0}$, for all $t \in \mathbb{R}$. Hence $A^{-\sigma} \widetilde{\mathfrak{y}}(t) \in \mathcal{M}$ is unique mild solution of the system $(1.1)-(1.3)$.

Theorem 3.2. Assume that $\left(\mathrm{H}_{1}\right)-\left(\mathrm{H}_{7}\right)$ are hold. Then the system (1.1) - (1.3) has a unique exponentially stable mild solution in $\mathcal{W P} \mathcal{A} \mathcal{P} \rho\left(\mathbb{R}, \mathbb{L}^{2}(\mathbb{P}, \mathbb{H})\right)$.

Proof. The existence and uniqueness followed by Theorem 3.1 and adopting the ideas developed in [11, Theorem 3.3], we come to the conclusion.

Remark 4. Consider the following equation with delay

$$
\begin{aligned}
{ }^{c} D_{t}^{\alpha} y(t)+A y(t) & =G(t, y(t-\eta))+F\left(t, y(t-\eta), \int_{-\infty}^{t} \mathcal{K}(t-s) g(s, y(s)) d s\right) \frac{d w(t)}{d t}, \\
& t_{0}<t \neq t_{i}, t \in \mathbb{R}, \\
y\left(t_{i}^{+}\right) & =y\left(t_{i}^{-}\right)+\mathcal{G}_{i}\left(y\left(t_{i}\right)\right), \quad i \in \mathbb{Z}, \\
y\left(t_{0}\right) & =y_{0},
\end{aligned}
$$

where $\eta$ is fix and $\eta \in \mathbb{R}^{+}$. Assume that $y=y_{1}+y_{2} \in \mathcal{W P} \mathcal{A} \mathcal{P}_{\rho}\left(\mathbb{R}, \mathbb{L}^{2}(\mathbb{P}, \mathbb{H})\right)$ where $y_{1} \in \mathcal{A} \mathcal{P}^{\mathfrak{p}}\left(\mathbb{R}, \mathbb{L}^{2}(\mathbb{P}, \mathbb{H})\right)$ and $\mathrm{y}_{2} \in \mathcal{P} \mathcal{A} \mathcal{P}_{\rho}\left(\mathbb{R}, \mathbb{L}^{2}(\mathbb{P}, \mathbb{H})\right)$. For given $\eta \in \mathbb{R}$, it is easy to show that $\mathrm{y}(\mathrm{t}-\eta) \in \mathcal{A} \mathcal{P}^{\mathrm{p}}\left(\mathbb{R}, \mathbb{L}^{2}(\mathbb{P}, \mathbb{H})\right)$.

For $\gamma>0$, we have

$$
\begin{aligned}
\frac{1}{\mu(\gamma, \rho)} \int_{-\gamma}^{\gamma} \mathbb{E}\left\|y_{2}(t-\eta)\right\|^{2} \rho(t) d t & =\frac{1}{\mu(\gamma, \rho)} \int_{-\gamma-\eta}^{\gamma-\eta} \mathbb{E}\left\|y_{2}(t)\right\|^{2} \rho(t+\eta) d t \\
& =\frac{\mu(\gamma+\eta, \rho)}{\mu(\gamma, \rho)} \times \frac{1}{\mu(\gamma+\eta, \rho)} \int_{-\gamma-\eta}^{\gamma-\eta} \mathbb{E}\left\|y_{2}(t)\right\|^{2} \frac{\rho(t+\eta)}{\rho(t)} \rho(t) d t .
\end{aligned}
$$


Since $\rho \in W_{T}$, then there exists $a_{0}>0$ such that $\frac{\rho(t+\eta)}{\rho(t)} \leq a_{0}, \frac{\rho(t-\eta)}{\rho(t)} \leq a_{0}$, For $\gamma>\eta$

$$
\begin{aligned}
\mu(\gamma+\eta, \rho) & =\int_{-\gamma-\eta}^{\gamma-\eta} \rho(t) d t+\int_{\gamma-\eta}^{\gamma+\eta} \rho(t) d t \\
& \leq \int_{-\gamma-\eta}^{\gamma-\eta} \rho(t) d t+\int_{-\gamma+\eta}^{\gamma+\eta} \rho(t) d t \\
& =\int_{-\gamma}^{\gamma} \frac{\rho(t-\eta)}{\rho(t)} \rho(t) d t+\int_{-\gamma}^{\gamma} \frac{\rho(t+\eta)}{\rho(t)} \rho(t) d t \leq 2 a_{0} \mu(\gamma, \rho) .
\end{aligned}
$$

Then by $\mathrm{y}_{2} \in \mathcal{P} \mathcal{A} \mathcal{P}_{\rho}\left(\mathbb{R}, \mathbb{L}^{2}(\mathbb{P}, \mathbb{H})\right)$, we get

$$
\frac{1}{\mu(\gamma, \rho)} \int_{-\gamma}^{\gamma} \mathbb{E}\left\|y_{2}(t-\eta)\right\|^{2} \rho(t) d t \leq \frac{2 a_{0}^{2}}{\mu(\gamma+\eta, \rho)} \int_{-\gamma-\eta}^{\gamma+\eta} \mathbb{E}\left\|y_{2}(t)\right\|^{2} \rho(t) d t \rightarrow 0,
$$

as $\gamma \rightarrow \infty$. Hence $y(t-\eta) \in \mathcal{W P} \mathcal{A} \mathcal{P}_{\rho}\left(\mathbb{R}, \mathbb{L}^{2}(\mathbb{P}, \mathbb{H})\right)$ for $\eta \in \mathbb{R}^{+}$. Thus the results of Theorem 3.1 holds for the system $(3.12)-(3.14)$.

\section{Example}

Now,we present an example, which do not aim at generality but indicate how our abstract result can be applied to concrete problem. Consider the stochastic fractional differential equation with impulsive effects

$$
\begin{aligned}
\frac{\partial^{\alpha}}{\partial t^{\alpha}} z(t, x)-\frac{\partial^{2}}{\partial x^{2}} z(t, x) & =G(t, x, z(t, x))+F\left(t, x, z(t, x), \int_{\infty}^{t} e^{-2(t-s)} g(s, x, z(t, x) d s) \frac{d w(t)}{d t}, t \in \mathbb{R},\right. \\
z\left(t_{i}^{+}, x\right) & =z\left(t_{i}^{-}, x\right)+\lambda_{i}\left(z\left(t_{i}, x\right)\right), \quad i \in \mathbb{Z}, x \in(0,1) \\
z(t, 0) & =z(t, 1)=0,
\end{aligned}
$$

where $t_{i}=i+\frac{1}{4}|\sin 3 i+\sin \sqrt{3} i|$ and assume that $\lambda_{i} \in \mathcal{W P} \mathcal{A} \mathcal{P}_{\rho}\left(\mathbb{Z}, \mathbb{L}^{2}(\mathbb{P}, \mathbb{H})\right), \rho \in W_{\mathrm{T}}$. Note that $\left\{t_{i}^{j}\right\}, i, j \in \mathbb{Z}$ are equipotentially almost periodic and $k=\inf _{i \in \mathbb{Z}}\left(t_{i+1}-t_{i}\right)>0$, for more details see $[14,21,24]$. Note that $w(t)$ represents a standard Wiener process on a complete probability space $\left(\Omega, \mathcal{F},\left\{\mathcal{F}_{t}\right\}_{t \geq 0}, \mathbb{P}\right)$, where $\left\{\mathcal{F}_{\mathrm{t}}\right\}_{\mathrm{t} \geq 0}$ is sigma algebra generated by $\{w(u)-w(v): u, v \leq t\}$.

Let $\mathbb{H}=\left(\mathrm{L}^{2}\left([0,1],\|\cdot\|_{\mathrm{L}^{2}}\right)\right.$ be a Hilbert space. Now define the operator

$$
A y(\xi):=-y^{\prime \prime}(\xi), \quad \xi \in(0,1), y \in D(A),
$$

where

$$
\mathrm{D}(\mathrm{A}):=\left\{\mathbb{H}^{2} \cap \mathbb{H}_{0}^{1}: \mathrm{y}^{\prime \prime} \in \mathbb{H}\right\} .
$$

Then, $A$ is the infinitesimal generator of analytic semigroup $\mathcal{S}(t)$ on $\mathbb{H}$. Now, we have $z_{n}(t)=$ $(2)^{\frac{1}{2}} \sin n \pi t, n=1,2,3, \ldots$, are the eigenfunction of $A$ corresponding to the eigenvalues $n \pi$. For $\sigma=\frac{1}{4}$ denote $D\left(A^{\frac{1}{4}}\right)$ by $\mathbb{L}^{2}\left(\mathbb{P}, \mathbb{H}_{\frac{1}{4}}\right)$ is a Banach space equipped with the norm

$$
\|y\|_{\frac{1}{4}}=\left\|A^{\frac{1}{4}} y\right\|, \quad y \in D\left(A^{\frac{1}{4}}\right) .
$$


Let $y(t) x=z(t, x), t \in \mathbb{R}, x \in[0,1]$, then

$$
F\left(t, y(t), \int_{-\infty}^{t} \mathcal{K}(t-s) g(s, y(s)) d s\right)=F\left(t, x, z(t, x), \int_{\infty}^{t} e^{-2(t-s)} g(s, x, z(t, x) d s) .\right.
$$

Now the system (4.1)-(4.3) can be reformulated in the abstract form of the system (1.1)-(1.3). Since $\mathcal{G}_{i}=\lambda_{i}$, then $\left(H_{6}\right)$ holds with $L_{\mathcal{G}}=\sup _{i \in \mathbb{Z}}\left\|\lambda_{i}\right\|$. We have the following result.

Theorem 4.1. Assume that the following assumptions hold:

(i) For $\rho \in W_{\mathrm{T}}, \mathrm{g} \in \mathcal{W} \mathcal{P} \mathcal{A P} \rho\left(\mathbb{R} \times \mathbb{L}^{2}\left(\mathbb{P}, \mathbb{H}_{\frac{1}{4}}\right), \mathbb{L}^{2}(\mathbb{P}, \mathbb{H})\right)$ and there exists a $\mathrm{L}_{\mathrm{g}}>0,0<\eta<1$ such that

$$
\mathbb{E}\left\|g\left(t_{1}, u_{1}\right)-g\left(t_{2}, u_{2}\right)\right\|^{2} \leq L_{g} \mathbb{E}\left(\left|t_{2}-t_{1}\right|^{n}+\left\|u_{1}-u_{2}\right\|_{\frac{1}{2}}^{2}\right.
$$

for all, $\left(\mathrm{t}_{i}, \mathrm{u}_{\mathrm{i}}\right) \in \mathbb{R} \times \mathbb{L}^{2}\left(\mathbb{P}, \mathbb{H}_{\frac{1}{4}}\right), \boldsymbol{i}=1,2$.

(ii) For $\rho \in W_{\mathrm{T}}, \mathrm{G} \in \mathcal{W} \mathcal{P} \mathcal{A} \mathcal{P}_{\rho}\left(\mathbb{R} \times \mathbb{L}^{2}\left(\mathbb{P}, \mathbb{H}_{\frac{1}{4}}\right), \mathbb{L}^{2}(\mathbb{P}, \mathbb{H})\right)$ and there exists $\mathrm{L}_{\mathrm{G}}>0,0<\eta<1$ such that

$$
\mathbb{E}\left\|G\left(t_{1}, u_{1}\right)-G\left(t_{2}, u_{2}\right)\right\|^{2} \leq L_{G}\left(\left|t_{2}-t_{1}\right|^{\eta}+\mathbb{E}\left\|u_{1}-u_{2}\right\|_{\frac{1}{2}}^{2}\right)
$$

for each $\left(\mathrm{t}_{i}, \mathfrak{u}_{i}\right) \in \mathbb{R} \times \mathbb{L}^{2}\left(\mathbb{P}, \mathbb{H}_{\frac{1}{4}}\right), i=1,2$.

(iii) For $\rho \in W_{\mathrm{T}}, \mathrm{F} \in \mathcal{W} \mathcal{P} \mathcal{A} \mathcal{P}_{\rho}\left(\mathbb{R} \times \mathbb{L}^{2}\left(\mathbb{P}, \mathbb{H}_{\frac{1}{4}}\right) \times \mathbb{L}^{2}\left(\mathbb{P}, \mathbb{H}_{\frac{1}{4}}\right), \mathbb{L}^{2}\left(\mathbb{P}, \mathbb{L}_{0}^{2}\right)\right)$ and there exists a $\mathrm{L}_{\mathrm{F}}>0$ such that

$$
\mathbb{E}\left\|\mathrm{F}\left(\mathrm{t}, \mathrm{u}_{1}, v_{1}\right)-\mathrm{f}\left(\mathrm{t}, \mathrm{u}_{2}, \mathrm{u}_{2}\right)\right\|^{2} \leq \mathrm{L}_{\mathrm{F}}\left(\left|\mathrm{t}_{2}-\mathrm{t}_{1}\right|^{\eta} \mathbb{E}\left\|\mathrm{u}_{1}-\mathrm{u}_{2}\right\|_{\frac{1}{2}}^{2}+\mathbb{E}\left\|v_{1}-v_{2}\right\|_{\frac{1}{2}}^{2}\right)
$$

for each $\left(\mathrm{t}_{i}, \mathrm{u}_{i}, v_{i}\right) \in \mathbb{R} \times \mathbb{L}^{2}\left(\mathbb{P}, \mathbb{H}_{\frac{1}{4}}\right) \times \mathbb{L}^{2}\left(\mathbb{P}, \mathbb{H}_{\frac{1}{4}}\right), i=1,2$.

Let us choose the constants $M_{\sigma}=1, \lambda=9, \mathrm{~L}_{\mathrm{G}}=1, \mathrm{~L}_{\mathrm{F}}=1, \mathrm{~L}_{\mathrm{g}}=1, \mathrm{~L}_{\mathcal{G}}=\frac{1}{2}$ and $\mathrm{N}_{\mathrm{O}}=\frac{1}{4}$, then we have

$$
\Delta:=3 \mathrm{M}_{\sigma}^{2}\left[4 \mathrm{~L}_{\mathcal{G}} \mathrm{N}_{0}^{2}\left(\frac{1}{M_{0}^{\sigma}}+\frac{1}{e^{\lambda}-1}\right)^{2}+\mathrm{L}_{\mathrm{G}} \frac{\Gamma^{2}\left(\frac{3}{4}\right)}{\lambda^{\frac{3}{2}}}+\mathrm{L}_{\mathrm{F}} \mathcal{N}_{\mathrm{O}}\left(1+\frac{\mathrm{L}_{g}}{4}\right) \frac{\sqrt{\pi}}{\lambda^{\frac{3}{2}}}\right]=0.69<1
$$

this implies that the system (4.1)-(4.3) has a unique piecewise square mean weighted pseudo almost periodic solution.

\section{Acknowledgment}

The authors would like to thank the editor and the reviewers for their valuable comments and suggestions. The work of the first author is supported by the "Ministry of Human Resource and Development, India under grant number:MHR-02-23-200-44". 


\section{References}

[1] Abbas S., Benchohra M. and N'Guérékata G.M.; Topics in fractional differential equations, Developments in Mathematics, Springer, New York (2012).

[2] Bahaj M. and Sidki O.; Almost periodic solutions of semilinear equations with analytic semigroups in Banach spaces, Elect. J. Diff. Equn. (2002), 1-11.

[3] Baleanu D., Diethelm K., Scalas E. and Trujillo J. J.; Fractional calculus models and numerical methods in: series on complexity, nonlinearity and Chaos, World Scientific, Boston, (2012).

[4] Bezandry P. and Diagana T.; Existence of almost periodic solutions to some stochastic differential equations, Applicable Anal., 7(2007), 819-827.

[5] Chérif F.; Pseudo almost periodic solutions of impulsive differential equations with delay, differ. Equ. Dyn. Syst. 22(1)(2014), 7391.

[6] Chang Y. K., Ma R. and Zhao Z. H.; Almost periodic solutions to a stochastic differential equation in Hilbert spaces, Results in Math., 63(2013), 435-449.

[7] Da Prato G. and Zabczyk J.; Stochastic equations in infinite dimensions, encyclopedia of mathematics and its applications,44, Cambridge University Press, Cambridge (1992).

[8] Diagana T.; Weighted pseudo almost periodic functions and applications, C. R. Acad. Sci. Paris, Ser. I 343 (2006), 643-646.

[9] Diagana T.; Stepanov-like pseudo-almost periodicity and its applications to some nonautonomous differential equations, Nonlinear Anal. 69 (2008), 4277-4285.

[10] El-Borai M. M. and Debbouche A.; Almost periodic solutions of some nonlinear fractional differential equations, Int. J. Contemp. Math. Sci. 4 (2009), 1373-1387.

[11] Guendouzi T. and Bousmaha L.; Almost periodic solutions for impulsive fractional stochastic evolution equations, Int. J. Anal. appl., 6(2014), 28-43.

[12] Kafash B., Lalehzari R., Delavarkhalafi A. and Mahmoudi E.; Application of Stochastic Differential System in Chemical Reactions via Simulation, MATCH Commun. Math. Comput. Chem. 71 (2014), 265-277.

[13] Liu J. and Zhang C.; Existence and stability of almost periodic solutions to impulsive stochastic differential equations, Cubo, 15(2013), 77-96.

[14] Liu J. W. and Zhang C. Y.; Composition of piecewise pseudo almost periodic functions and applications to abstract impulsive differential equations, Adv. Diff. Eqn. (2013), 1-21. 
[15] Machado J. A. T.; Analysis and design of fractional order disital control system, Syst. Anal. Model. Simul. 27 (2-3) (1997), 107-122.

[16] Miller K. S. and Ross B.; An Introduction to the fractional calculus and differential equations, John Wiley, New York (1993).

[17] Pazy A.; Semigroups of linear operators and applications to partial differential equations, Springer-Verlage, New Tork, (1983).

[18] Podlubny I.; Fractional differential equations, Academic Press, New York (1999).

[19] Sakthivel R., Revathi P. and Renc Y.; Existence of solutions for nonlinear fractional stochastic differential equations. Nonlinear Anal. 81 (2013), 70-86.

[20] Sakthivel R., Suganya S. and Anthoni S. M.; Approximate controllability of fractional stochastic evolution equations, Comput. Math. Appl. 63 (2012), 660-668.

[21] Samoilenko A. M. and Perestyuk N. A.; Impulsive differential equations, vol. 14, World Scientific, Singapore, (1995).

[22] Stamov G.T. and Alzabut J.O.; Almost periodic solutions for abstract impulsive differential equations, Nonlinear Anal. TMA., 72 (2010), 2457-2464.

[23] Xia Z. and Wang D.; Piecewise weighted pseudo almost periodic solutions of impulsive integro-differential equations via fractional operators, Elect. J. Diff. Equn. 185(2015), 1-18.

[24] Xia Z.; Pseudo almost periodic mild solution of nonautonomous impulsive integro differential equations, Mediterr. J. Math. DOI 10.10007/s00009-015-0532-4.

[25] Zhang C. Y.; Pseudo almost periodic solutions of some differential equations, J. Math. Anal. Appl. 181 (1994), 62-76 .

[26] Zhang C. Y.; Pseudo almost periodic solutions of some differential equations, II, J. Math. Anal. Appl. 192 (1995), 543-561.

[27] Zhinan X.; Pseudo almost periodicity of fractional integro-differential equations with impulsive effects in Banach spaces, Czechoslovak Math. J. 67(142) (2017), no. 1, 123-141.

[28] Zhou Y. and Jiao F.; Nonlocal Cauchy problem for fractional evolution equations, Nonlinear Anal.: Real World Appl. 11 (2010), 4465-4475.

[29] Zhou Y. and Jiao F.; Existence of mild solutions for fractional neutral evolution equations, Comput. Math. Appl. 59 (2010), 1063-1077. 